\title{
Wie oft und zu welchen Themen werden schulinterne Lehrkräftefortbildungen angeboten? Empirische Befunde einer vergleichenden Programmanalyse
}

\author{
Jenny Kuschel iD $\cdot$ Eric Richter $\cdot$ Rebecca Lazarides $・$ Dirk Richter
}

Eingegangen: 21. Dezember 2020 / Überarbeitet: 8. Juni 2021 / Angenommen: 8. Juni 2021 / Online publiziert: 12. Juli 2021

(C) Der/die Autor(en) 2021

Zusammenfassung Mit dem Systemwandel von der Input- zur Outputsteuerung im Bildungswesen ging eine Erhöhung einzelschulischer Gestaltungsspielräume einher. Einen Bereich erweiterter schulischer Selbstständigkeit und Eigenverantwortung stellen schulinterne Fortbildungen dar. Obwohl schulinternen Fortbildungen eine hohe Bedeutung für die qualitativ hochwertige Entwicklung der Einzelschule zugeschriebenen wird, liegen bislang nur wenige Forschungsbefunde zur Nutzung und den Themen schulinterner Fortbildungsangebote vor. Die vorliegende Studie verfolgt daher das Ziel, das schulinterne Fortbildungsangebot hinsichtlich des Angebotsumfangs, der angebotenen Themen und des Zusammenhangs zwischen Schulmerkmalen und der Nutzung unterschiedlicher schulinterner Fortbildungsthemen zu untersuchen. Hierfür wurden Daten aus der elektronischen Datenbank für Lehrkräftefortbildungen des Landes Brandenburg aus den Schuljahren 2016/17 und 2017/18 ausgewertet. Die Ergebnisse zeigen, dass die Mehrheit der Schulen im Untersuchungszeitraum keine staatlichen schulinternen Fortbildungen durchführen. Darüber hinaus verdeutlichen Ergebnisse eines Chi-Quadrat-Tests zum Vergleich des schulinternen und -externen Fortbildungsangebots, dass sich die inhaltlichen Schwerpunkte in den Fortbildungsformaten substantiell unterscheiden. Schließlich zeigen die Ergebnisse von Regressionsanalysen, dass ein Zusammenhang zwischen Merkmalen des Lehrkräftekollegiums, Merkmalen der Schülerinnen und Schüler sowie Ergebnissen aus Vergleichsarbeiten und der Wahl bestimmter schulinterner Fortbildungsthemen besteht. Die Befunde geben damit erstmalig Einblicke in die Angebotsstruktur und Nutzung schulinterner Fortbildungen im Zusammenhang mit Schulmerkmalen. Mögliche Implikationen für eine stärkere Nutzung des schulinternen Fortbildungsangebots werden diskutiert.

Jenny Kuschel $(\varangle) \cdot$ Eric Richter $\cdot$ Rebecca Lazarides $\cdot$ Dirk Richter Universität Potsdam, Karl-Liebknecht-Straße 24-25, 14476 Potsdam, Deutschland E-Mail: jkuschel@uni-potsdam.de 
Schlüsselwörter Lehrkräftefortbildung · Professionalisierung ·

Personalentwicklung

\title{
How often and on what topics are in-school professional development programs offered? Empirical findings of a comparative program analysis
}

\begin{abstract}
The change from an input- to an output-oriented model of educational governance provided schools with more autonomy. This includes the opportunity to develop their school-based professional development program. These kinds of programs enable schools to cultivate the competencies of their teaching staff based on the schools' priorities. However, only a few studies have focused on research in the field of in-school professional development activities. Therefore, the present study examines in-school professional development activities with regard to the number of courses, their topics and the relationship between school characteristics and different in-school professional development topics. For this purpose, data from the electronic database on teacher's professional development activities in the state of Brandenburg in the years 2016/17 and 2017/18 were evaluated. The results show that the majority of schools did not offer any in-school professional development during the respective time period. In addition, results of a chi square test shows that the content of in-school and school-external professional development activities differs. Finally, results of regression analyses confirmed relationships between the number of courses provided on the one hand and the characteristics of the teaching staff, the composition of the student body and results of statewide competency tests on the other. The findings provide insights into the offerings of in-school professional development activities and their relationship to school characteristics. Possible implications for increasing the use of in-school professional development activities are discussed.
\end{abstract}

Keywords Professional development $\cdot$ Staff development $\cdot$ School development

Ergebnisse der Fortbildungsforschung zeigen, dass Lehrkräftefortbildungen das Potenzial besitzen, die professionelle Entwicklung von Lehrkräften zu unterstützen (Darling-Hammond et al. 2017). Lehrkräften wird aus diesem Grund in ganz Deutschland ein umfangreiches staatliches Fortbildungsangebot unterbreitet, welches sie im Rahmen ihrer dienstlichen Tätigkeiten in Anspruch nehmen können (DVLfB 2018). Während in bisherigen Studien vor allem die Nutzung und Wirksamkeit schulexterner Fortbildungsangebote im Mittelpunkt standen (z.B. Rzejak und Lipowsky 2020; Richter und Richter 2020), stellen schulinterne Fortbildungen ein Forschungsdesiderat dar (Daschner 2019).

Die vergleichsweise wenigen Forschungsarbeiten zu schulinternen Fortbildungen überraschen, denn diese Art der Lehrkräftefortbildung werden als bedeutsam für die Weiterentwicklung von Schule betrachtet (Wenzel und Wesemann 1990). Erkenntnisse über die Nutzung und das Angebot schulinterner Fortbildungen könnten deshalb ein besseres Verständnis der Funktionsweise schulinterner Fortbildungen 
als Mittel der Schulentwicklung ermöglichen (Wenzel und Wesemann 1990). In länderspezifischen Regelungen ist festgelegt, dass die Planung schulinterner Fortbildungen unter Berücksichtigung der internen Qualifikationsbedarfe der Einzelschule (z.B. Eckpunkte des Schulprofils oder Leistungen der Schülerinnen und Schüler) durch die Schulleitung erfolgen soll (DVLfB 2018). Untersuchungen von van Ackeren et al. (2013) zeigen, dass sich Schulleitungen im Kontext von Schulentwicklungsprozessen überwiegend auf schulinterne Datenquellen (z. B. Schülerfeedback, kollegiale Hospitationen) beziehen und auf dieser Grundlage Maßnahmen ergreifen. Bislang liegen jedoch noch keine Untersuchungen dazu vor, inwiefern bei der Planung schulinterner Fortbildungen Merkmale der Einzelschule eine Rolle spielen. Es stellt sich deshalb die Frage, inwiefern Schulen mit unterschiedlichen Voraussetzungen auch unterschiedliche schulinterne Fortbildungsangebote nutzen.

Die vorliegende Arbeit geht der Frage nach, in welchen Umfang und zu welchen Themen Schulen interne Fortbildungen anbieten, inwiefern es sich vom externen Fortbildungsangebot unterscheidet und inwieweit Merkmale der Schule im Zusammenhang mit der Nutzung unterschiedlicher Themen in schulinternen Fortbildungen stehen. Zur Beantwortung der Fragestellung werden zunächst Merkmale schulinterner Fortbildungen beschrieben und deren Funktion für Schulentwicklungsprozesse verdeutlicht. In einem weiteren Schritt werden anhand des Modells von Qualität und Qualitätssicherung in Schule und Unterricht (Ditton 2000) die strukturellen, personellen und sozialen Bedingungen einer Schule sowie die Leistungen der Schülerinnen und Schüler als bedeutsame Prädiktoren für die Auswahl spezifischer Themen schulinterner Fortbildungen im Rahmen von schulischer Personalentwicklung identifiziert. Anschließend wird mittels Regressionsanalysen überprüft, inwiefern diese Prädiktoren im Zusammenhang mit dem schulinternen Fortbildungsangebot stehen. Darüber hinaus erfolgt mit Hilfe eines Chi-Quadrat-Tests ein Vergleich des schulinternen und -externen Fortbildungsangebots, um mögliche thematische Unterschiede zwischen den Fortbildungsformaten zu untersuchen. Abschließend werden die Ergebnisse vor dem Hintergrund bestehender Befunde der Fortbildungsforschung diskutiert.

\section{Schulinterne Fortbildung als Instrument der Schulentwicklung}

Seit Beginn der 1990er lässt sich für das Bildungswesen ein Systemwandel konstatieren, der mit dem Begriff „Neue Steuerung“ bezeichnet wird und mit einem höheren Ausmaß an Schulautonomie einhergeht (Altrichter und Maag Merki 2016). Diese veränderte Strategie politisch-administrativer Steuerung von Schule bewirkte unter anderem eine „Umverteilung von Entscheidungsrechten von übergeordneten Verwaltungseinheiten zu den Einzelschulen“ (Altrichter und Maag Meerki 2016, S. 21) und führte damit zu einer Erhöhung der Selbstständigkeit und Eigenverantwortung der Schulen. Schulinterne Lehrkräftefortbildungen (SchiLF) stellen im Zuge dieser Entwicklung einen zentralen Bereich schulischer Autonomie dar (Altrichter et al. 2016) und wurden bereits in den 1980er-Jahren zunehmend von Schulen genutzt (Basold 2010). Mit der Erhöhung einzelschulischer Gestaltungsspielräume war die Annahme verbunden, dass Schulen sich dann besonders gut strukturell entwickeln 
können, wenn sie auf ihre Bedarfe ausgerichtete Maßnahmen identifizieren können, um auf Problemlagen und Herausforderungen zu reagieren (Altrichter et al. 2016). Aus diesem Grund werden SchiLF für die Schulentwicklung als besonders relevant angesehen (Basold 2010). Schulentwicklung wird in diesem Zusammenhang als eine bewusste, absichtsvolle und systematische Weiterentwicklung der Einzelschule durch deren Mitglieder in Form eines fortlaufenden Prozesses verstanden (Dedering 2012).

Schulinterne Fortbildungen zielen als Instrument der Schulentwicklung auf die Erhöhung von Schulqualität (Tillmann 2011), die als mehrdimensionales Konstrukt anhand verschiedener Merkmale beschrieben werden kann (Ditton und Müller 2015). Ditton (2000) beschreibt beispielsweise in seinem heuristischen Modell von Qualität und Qualitätssicherung in Schule und Unterricht Annahmen über die Zusammenhänge zwischen schulstrukturellen Voraussetzungen, schulischen Prozessmerkmalen und ihren Wirkungen auf Bildungsergebnisse von Schülerinnen und Schülern.

$\mathrm{Zu}$ den schulbezogenen Voraussetzungen zählen unter anderem die strukturellen, finanziellen, personellen und sozialen Bedingungen einer Schule. Darüber hinaus gehören auch die intendierten Bildungsziele sowie die Leistungen, Einstellungen und Haltungen von Schülerinnen und Schülern zu den Voraussetzungen für Prozesse in Schule und Unterricht. Die schulbezogenen Voraussetzungen stehen in Wechselwirkung mit der Qualität der Schule (Schulkultur, Management, Kooperationen und Personalentwicklung). Schulbezogene Voraussetzungen stehen aber auch in Verbindung mit Qualitätsmerkmalen des Unterrichts, wie z.B. der Adäquatheit der Lehrinhalte. Prozessbezogene Qualitätsmerkmale der Schule und des Unterrichts als auch die schulbezogenen Voraussetzungen bedingen gemeinsam die Qualität schulischer Ergebnisse, zu denen Ditton (2000) schulische Leistungen sowie Einstellungen und Haltungen der Schülerinnen und Schüler zählt, die wiederum langfristig den beruflichen Erfolg und die gesellschaftliche Teilhabe prägen (Abb. 1).

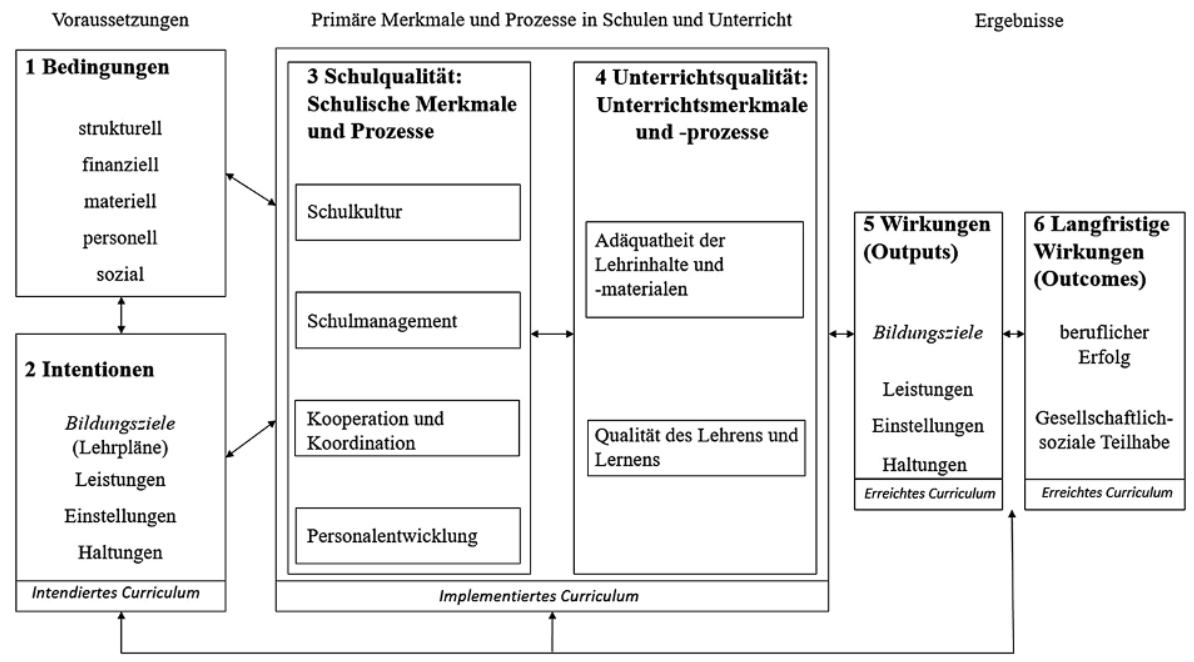

Abb. 1 Modell von Qualität und Qualitätssicherung in Anlehnung an Ditton (2000) 
Nach dem Modell von Ditton (2000) stellt SchiLF ein Prozessmerkmal der Schulqualität dar. Den Annahmen des Modells folgend, stehen SchiLF im Zusammenhang mit den schulbezogenen Voraussetzungen, also den strukturellen, personellen und sozialen Bedingungen. Als strukturelle Bedingungen werden die Schulart und die Schulgröße beschrieben (Ditton 2000), für die im Modell eine Verbindung zum Prozessmerkmal Personalentwicklung konstatiert wird. Bislang ist der Zusammenhang zwischen strukturellen Bedingungen und Prozessen der Personalentwicklung nur unzureichend erforscht (Thillmann et al. 2015), jedoch können Erkenntnisse hierüber einerseits einen Beitrag zur Klärung von Unterschieden in der Qualität von Schulen leisten und andererseits Hinweise auf unterschiedliche schulische Unterstützungsbedarfe geben. Die wenigen vorliegenden Forschungsbefunde zum Zusammenhang zwischen schulischen strukturellen Bedingungen und Personalentwicklungsprozessen liefern kein eindeutiges Bild. Appius et al. (2012) berichten beispielsweise in ihrer Studie zur Personalentwicklung an deutschen und schweizerischen Schulen, dass die Umsetzung von Personalförderungsmaßnahmen im Zusammenhang mit der Größe des Kollegiums steht. In großen Schulen werden in dieser Studie mehr Personalentwicklungsmaßnahmen realisiert als in Schulen mit einem kleineren Kollegium. Es bleibt jedoch offen, wie dieser Zusammenhang erklärt werden kann. Bach et al. (2020) kommen in ihrer Studie zum Zusammenhang zwischen schulischer Personalentwicklung und der Entwicklung unterrichtsbezogener Kompetenzen der Lehrkräfte hingegen zu dem Ergebnis, dass mit zunehmender Schulgröße weniger Personalentwicklungsmaßnahmen durchgeführt werden. Jedoch berücksichtigten Bach et al. (2020) in ihrer Studie nur individuelle Personalentwicklungsmaßnahmen für einzelne Lehrkräfte (Unterrichtshospitationen durch die Schulleitung, Zielvereinbarungsgespräche) und nicht die Nutzung von SchiLF. Die Forschungsergebnisse weisen somit darauf hin, dass strukturelle schulische Bedingungen im Zusammenhang mit der Durchführung von Personalentwicklungsmaßnahmen wie schulinternen Fortbildungen stehen, jedoch ist die Befundlage hierzu nicht eindeutig.

Neben den strukturellen Bedingungen werden in Dittons Modell personelle Bedingungen unter den Voraussetzungen von Schulen aufgeführt, die in einem wechselseitigen Verhältnis zur Personalentwicklung der Schule stehen. Hierunter können beispielsweise Merkmale des Lehrkräftekollegiums, etwa die Altersstruktur oder der Anteil an Lehrkräften im Quer- und Seiteneinstieg, gefasst werden. Im Allgemeinen verfolgt schulische Personalentwicklung das Ziel, Lehrkräfte ausgehend von ihren Fähigkeiten und Neigungen zur Bewältigung beruflicher Anforderungen zu befähigen (Mentzel 2001). Für die Bewältigung der Anforderungen des Lehrberufs ist die Entwicklung professioneller Kompetenzen entscheidend (Baumert und Kunter 2006), die unter anderem durch die Nutzung von Lerngelegenheiten im Lehramtsstudium erworben werden können (Felbrich et al. 2008).

Lehrkräfte im Quer- und Seiteneinstieg, die kein traditionelles Lehramtsstudium absolviert haben und über einen alternativen Zugang in den Schuldienst eingemündet sind, stellen vor diesem Hintergrund eine besondere Gruppe innerhalb eines Lehrkräftekollegiums dar. Verschiedene Studien zeigen, dass Lehrkräfte im Querund Seiteneinstieg über weniger pädagogisches Wissen verfügen als traditionell ausgebildete Lehrkräfte (z.B. Lucksnat et al. 2020). Die Forschungsergebnisse deuten darauf hin, dass systematische Unterschiede in den Kompetenzen eines Kollegi- 
ums vorliegen können. Es wäre demnach anzunehmen, dass ein hoher Anteil an Lehrkräften im Quer- und Seiteneinstieg im Kollegium besondere Personalentwicklungsmaßnahmen in Form von Fortbildungen in der Schule erfordern könnte, um Kompetenzunterschiede auszugleichen. Bisher ist jedoch offen, inwiefern Schulen den Fortbildungsbedarf der nicht-traditionell ausgebildeten Lehrkräfte mit Hilfe von SchiLF bearbeiten und welche thematischen Fortbildungsangebote für Schulen mit einem hohen Anteil an Lehrkräften im Quer- und Seiteneinstieg relevant sind.

Weiterhin werden im Modell von Ditton (2000) soziale Bedingungen unter den Voraussetzungen der Schulen aufgeführt, welche ebenfalls in Verbindung mit Maßnahmen der schulischen Personalentwicklung stehen. Unter diesem Begriff lassen sich Merkmale der Schülerinnen und Schüler hinsichtlich verschiedener Aspekte verstehen, z.B. der sozioökonomische Status oder ihr sonderpädagogischer Förderbedarf. Insbesondere die Inklusion von Schülerinnen und Schülern mit sonderpädagogischen Förderbedarf an allgemeinbildenden Schulen stellt Lehrkräfte vor neue Chancen und Herausforderungen hinsichtlich der Unterrichtsgestaltung und erfordert eine Erweiterung ihrer professionellen Kompetenzen, u. a. im Hinblick auf fachdidaktisches und (sonder-)pädagogisches Fachwissen (Werding und Schinnenburg 2016). Es stellt sich die Frage, ob Schulen durch ein Angebot an SchiLF in diesem Themenbereich den Fortbildungsbedarf ausreichend bedienen können.

Darüber hinaus stellt Ditton (2000) in seinem Modell einen Bezug zwischen Personalentwicklung und den Bildungsergebnissen in Form von Leistungen der Schülerinnen und Schüler her. Im Rahmen des Bildungsmonitoring sind Ergebnissen aus Vergleichsarbeiten (VERA) von besonderer Bedeutung, da hierfür deutschlandweite Standards gelten und dadurch eine Vergleichbarkeit der Ergebnisse hergestellt wird (KMK 2004). Die Ergebnisse der Vergleichsarbeiten sollen den Lehrkräften eine Rückmeldung dazu geben, in welchen Bereichen ihre Schülerinnen und Schüler die Bildungsstandards erreichen und wo weitere Unterstützung notwendig ist. Indem Lehrkräfte die Rückmeldungen für die Weiterentwicklung des Unterrichts nutzen, wird eine datenbasierte Schulentwicklung angestrebt (Maier et al. 2012). Es stellt sich damit die Frage, inwiefern Ergebnisse aus Vergleichsarbeiten für die schulische Personalentwicklung von Bedeutung sind und wie diese für die Planung von SchiLF genutzt werden.

Unter Zuhilfenahme des Modells von Ditton (2000) kann somit angenommen werden, dass schulische Merkmale im Zusammenhang mit der Nutzung von SchiLF stehen. Aufgrund der Komplexität des Modells konnten bislang nur Teilaspekte anhand empirischer Daten überprüft werden (Ditton und Müller 2015). Deshalb ist bisher offen, wie sich der Zusammenhang zwischen den Voraussetzungen einer Schule und der Nutzung von SchiLF gestaltet. Wie Schulen SchiLF organisieren und welche Themen hierbei von Bedeutung sind, wird im folgenden Abschnitt beschrieben.

\section{Organisation und Nutzung schulinterner Fortbildungen}

In allen Bundesländern bestehen Regelungen zur Durchführung von SchiLF. Diese sind unter anderem in den Landesbeamtengesetzen, Lehrerbildungsgesetzen und eigenen Erlassen festgeschrieben (KMK 2017). In Brandenburg ist beispielsweise 
in der Verwaltungsvorschrift über die Fortbildung der Lehrkräfte an Schulen in öffentlicher Trägerschaft (VV-Lehrkräftefortbildung, 2015) festgelegt, dass jedes Jahr unter Berücksichtigung des Schulprofils, der erreichten Leistungen der Schülerinnen und Schüler, der Ergebnisse der Schulvisitation und weiterer Evaluationsergebnisse sowie des Entwicklungsbedarfs infolge bildungspolitischer Veränderungen ein interner Fortbildungsplan erstellt werden soll, in dem die Fortbildungsschwerpunkte festgelegt sind (VV-Lehrkräftefortbildung § 5 Absatz 1, 2, 2015). Darüber hinaus ist die professionelle Weiterentwicklung als Aufgabe von Lehrkräften definiert (KMK 2000). SchiLF stellen neben externen Fortbildungen eine Möglichkeit zur Entwicklung ihrer professionellen Kompetenzen dar.

Die Organisation und Durchführung der SchiLF liegt in der Verantwortung der Einzelschule. Sie verfügt über umfangreiche inhaltliche und organisatorische Freiheit hinsichtlich der Auswahl und Gestaltung der SchiLF. Zudem kann die Schule darüber entscheiden, ob die Veranstaltung vom eigenen Kollegium organisiert und von ausgewählten Lehrkräften durchführt wird oder externe Referenteninnen und Referenten eingeladen werden (Wenzel und Wesemann 1990). Auch die inhaltliche Schwerpunktsetzung der SchiLF obliegt der Schule. Sie basiert auf einer schulinternen Bedarfsanalyse, die durch die Schulleitung oder andere dazu beauftragte Lehrkräfte, wie z.B. der/die Fortbildungsbeauftragte, in einem schulinternen Fortbildungsplan festgehalten wird. Schulen sind bundesweit verpflichtet, die Inhalte der einzelnen Veranstaltungen dort verbindlich festzulegen (Richter 2016). Bisher ist allerdings wenig darüber bekannt, wie Schulen dabei vorgehen und welche Informationen bei der Erstellung der Pläne berïcksichtigt werden.

Im Gegensatz zu externen Lehrkräftefortbildungen, welche regional durch verschiedene staatliche und nichtstaatliche Fortbildungsanbieter durchgeführt werden und in der Regel von einzelnen Lehrkräften einer Schule besucht werden, sind SchiLF darauf ausgerichtet, Lernprozesse des gesamten Kollegiums oder einzelner Gruppen anzustoßen (Wenzel und Wesemann 1990). Dadurch haben Schulen mit Hilfe von SchiLF die Möglichkeit, selbstständig auf schulinterne Problemlagen zu reagieren und aktiv gemeinschaftliche Lerngelegenheiten zu schaffen. Bislang ist jedoch unklar, inwiefern Schulen ihre Gestaltungsspielräume hinsichtlich der Planung und Durchführung von SchiLF für Schulentwicklung nutzen.

Informationen zum Umfang des schulinternen Fortbildungsangebots und den Themen von SchiLF existieren bislang nur für wenige Bundesländer. Im Hinblick auf den Angebotsumfang wurden beispielsweise in Brandenburg im Jahr 2014 bei einer Gesamtanzahl von 853 Schulen (Amt für Statistik Berlin-Brandenburg 2015) insgesamt 385 und im Jahr 2015 insgesamt 169 Veranstaltungen durchgeführt (DVLfB 2018). In Thüringen fanden in 2016 insgesamt 688 Veranstaltungen verteilt auf 834 staatliche Schulen statt (DVLfB 2018). Die Befunde zeigen, dass sich der Umfang des SchiLF-Angebots zwischen den Bundesländern unterscheidet. Zudem scheint es einen gewissen Anteil an Schulen zu geben, die keine SchiLF anbieten. Die bestehenden Befunde deuten somit darauf hin, dass sich Schulen in der Nutzung von SchiLF stark unterscheiden. Unklar ist jedoch, mit welchen Merkmalen das unterschiedliche Nutzungsverhalten zusammenhängt.

Nicht nur die quantitative Nutzung schulinterner Fortbildungen ist bislang wenig erforscht, sondern auch die in der Fortbildung adressierten Themen. Lediglich für 
die Bundesländer Brandenburg und Thüringen liegen Angaben zu den Themen von SchiLF vor. Interne Fortbildungen werden hier zu einem weiten Themenspektrum zu Schul- und Unterrichtsentwicklung aber auch zur Gesundheitsförderung angeboten. In Thüringen lag der Schwerpunkt in 2016 auf Fachfortbildungen (35\%), gefolgt von SchiLF zu Schulentwicklung (30\%). Eher selten wurden Veranstaltungen zu den Themen Inklusion ( $8 \%$ ) sowie Förderung und Begabung $(5 \%)$ angeboten (DVLfB 2018). Pasternack et al. (2017) berichten für Brandenburg, dass 2015 SchiLF zu den Kategorien schulische Organisationsentwicklung, unterrichts- und schülerbezogene Themen, außerunterrichtlichen Themen sowie lehrerbezogener Persönlichkeitsentwicklung und Professionalisierung durchgeführt wurden. Für diese Daten aus Brandenburg liegen jedoch keine Angaben zu dem jeweiligen Anteil an Fortbildungen pro Thema vor.

Die Analyse der schulinternen Fortbildungsthemen verdeutlicht, dass ein weites Themenspektrum angeboten wird. Bisher wurde die Frage nach Gemeinsamkeiten und Unterschieden in schulinternen und schulexternen Fortbildungsangeboten nicht untersucht. Die vorliegende Studie greift diesen Aspekt auf, um der Frage nachzugehen, inwiefern schulinterne Fortbildungen inhaltlich ein breiteres Spektrum anbieten, um möglichst verschiedene Bedarfe der Einzelschule effizient zu adressieren. Da sich SchiLF im Gegensatz zu externen Fortbildungsangeboten in der Regel an ganze Kollegien einer Schule richtet und die Gesamtheit der Bedarfe auf Schulebene adressiert, kann angenommen werden, dass sich die Themen vom externen Fortbildungsangebot unterscheiden. Pasternack et al. (2017) berichten dahingehend, dass schulintern wenige Fortbildungen zu fachdidaktischen Themen angeboten werden, da SchiLF vor allem Inhalte abdecken, die für alle Lehrkräfte eines Kollegiums relevant sind (z. B. Umgang mit Heterogenität). Allerdings erfolgte bisher keine differenzierte Analyse zu den Unterschieden zwischen den beiden Fortbildungsformaten (Daschner 2019).

Eine weitere offene Frage ist, inwiefern Zusammenhänge zwischen schulischen Rahmenbedingungen (z. B. Merkmale der Schülerinnen und Schüler) und der Auswahl von Fortbildungsthemen bestehen. Zum Beispiel gibt es Hinweise darauf, dass die Nutzung von Lehrkräftefortbildungen von der Schulart abhängig sein könnte. So zeigen etwa die Ergebnisse des IQB-Bildungstrends 2018, dass Lehrkräfte unterschiedlicher Schularten Fortbildungen mit unterschiedlichen Inhalten besuchen (Richter et al. 2019). Lehrkräfte an Gymnasien nehmen häufiger an Fortbildungen $\mathrm{zu}$ fachlichen und curricularen Themen teil und Lehrkräfte an nichtgymnasialen Schulen nutzen vermehrt Fortbildungen zu pädagogischen Themen wie Inklusion/ Integration, Umgang mit Störungen und Gewaltprävention besuchten (Richter et al. 2019). Vor dem Hintergrund, dass sich Merkmale der Schülerinnen und Schüler in Anhängigkeit von der Schulart unterscheiden können (z. B. Baumert et al. 2009), können die Ergebnisse des IQB-Bildungstrends 2018 dahingehend gedeutet werden, dass schulischen Rahmenbedingungen wie zum Beispiel die Merkmale der Schülerinnen und Schüler, unterschiedliche Herausforderungen für Lehrkräfte mit sich bringen. Da Schulen mit Hilfe von SchiLF die Möglichkeit haben, selbstständig auf schulinterne Ausgangslagen zu reagieren, ist anzunehmen, dass diese Themen im Rahmen von SchiLF aufgegriffen werden könnten. 


\section{Fragestellungen der Studie}

Zusammengefasst ist auf Grundlage des theoretischen Modells von Ditton (2000) anzunehmen, dass schulische Merkmale mit der Nutzung von SchiLF im Zusammenhang stehen. Allerdings gibt es nur vereinzelte deskriptive Befunde dazu, wie unterschiedlich Schulen SchiLF nutzen (Pasternack et al. 2017; DVLfB 2018). Die Frage, welche Themen für SchiLF besonders relevant sind und wie diese in Verbindung zu Merkmalen der Schule stehen, kann somit auf Basis bisheriger Forschung nicht beantwortet werden. Kenntnisse über das Ausmaß der Nutzung und die Inhalte von SchiLF sowie über Zusammenhänge zu Schulmerkmalen sind für die Fortbildungsforschung von Bedeutung, da sie Hinweise dahingehend liefern können, inwiefern Schulen Fortbildungsangebote zur Unterstützung im Rahmen von Schulentwicklungsprozessen nutzen.

Vor diesem Hintergrund soll mit der vorliegenden Studie ein Beitrag zur Klärung des Angebotsumfangs und den Inhalten von SchiLF im Vergleich zu externen Fortbildungsangeboten geleistet werden. Außerdem soll untersucht werden, inwieweit ein Zusammenhang zwischen Merkmalen der Schule und den von der Schule gewählten Themen für interne Fortbildungen bestehen. Im Anschluss an die vorangestellten Forschungsergebnisse leiten sich die folgenden drei Fragen ab:

Forschungsfrage 1 In welchem Umfang werden SchiLF an Schulen angeboten?

Forschungsfrage $2 \mathrm{Zu}$ welchen Themen werden SchiLF angeboten und bestehen hierbei Unterschiede zwischen dem internen und externen Fortbildungsangebot hinsichtlich der Häufigkeiten der angebotenen Themen?

Forschungsfrage 3 Inwieweit besteht ein Zusammenhang zwischen Merkmalen der Schule und der Auswahl bestimmter Themen von SchiLF?

\section{Datengrundlage}

Die Daten zum Angebot der schulinternen und -externen Fortbildungen basieren auf Angaben der Fortbildungsdatenbank des Landes Brandenburg und bilden die Grundlage für die Analysen. In der Datenbank sind die Daten zum Fortbildungsangebot verschiedener staatlicher Anbieter hinterlegt. Zu den staatlichen Anbietern gehören im Land Brandenburg die vier Staatlichen Schulämter, das Beratungs- und Unterstützungssystem für Schulen und Schulaufsicht (BUSS), das Landesinstitut für Schule und Medien Berlin-Brandenburg (LISUM) sowie das Ministerium für Bildung, Jugend und Sport (MBJS) (Missal 2019). Angebote nichtstaatlicher Anbieter, z.B. von Verlagen, sind in der Datenbank nicht verzeichnet und können daher im Rahmen der vorliegenden Arbeit nicht berücksichtigt werden.

Für das externe Fortbildungsangebot liegen Informationen aus dem Schuljahr 2016/17 (01.08.2016-31.07.2017) vor. Für schulinterne Fortbildungen liegt ebenfalls ein Datensatz für das Schuljahr 2016/17 und darüber hinaus für das Jahr 2017/18 (01.08.2017-31.07.2018) vor. Für die Bearbeitung der Forschungsfragen 1 und 3 
werden die aus den beiden Schuljahren 2016/17 und 2017/18 zur Verfügung stehenden Daten zu schulinternen Fortbildungen genutzt, um eine möglichst große Stichprobe zu erhalten und damit eine höhere Präzision der Schätzungen und eine größere statistische Power zu erzielen. Für den Vergleich des schulinternen und -externen Fortbildungsangebotes (Forschungsfrage 2) können nur Daten aus einem Schuljahr 2016/17 berücksichtigt werden, da für das externe Fortbildungsangebot nur Informationen aus diesem Schuljahr vorliegen.

In den Datensätzen zum schulinternen und -externen Fortbildungsangebot liegen für jede verzeichnete Fortbildung der Veranstaltungstitel vor. Die Datensätze enthalten sowohl Einzelveranstaltungen als auch Fortbildungsreihen. Fortbildungsreihen bestehen aus mehreren thematisch zusammengehörigen Veranstaltungen, die in der Regel über einen längeren Zeitraum verteilt stattfinden. Fortbildungen in diesem Format werden für die Analyse der Themenschwerpunkte im Datensatz als ein Fortbildungsangebot betrachtet.

Die Daten zum schulexternen Fortbildungsangebot wurden in Anlehnung an Richter et al. (2020) anhand nachfolgender Kriterien aufbereitet. Zunächst wurde der ursprüngliche Datensatz mit $N=4910$ Einträgen bereinigt. Hierbei wurden nur von den staatlichen Schulämtern durchgeführte Veranstaltungen berücksichtigt, da diese die Angebote für reguläre Lehrkräfte organisieren. Weiterhin werden alle Weiterbildungs- und Qualifizierungsmaßnahmen sowie Beratungsveranstaltungen und Regionalgruppentagungen ausgeschlossen, da diese nicht der Definition einer Lehrkräftefortbildung im engeren Sinne entsprechen. Der bereinigte Datensatz umfasst $N=932$ externe Fortbildungen.

Die Daten zum schulinternen Fortbildungsangebot beinhalten neben dem Veranstaltungstitel eine Angabe zum Veranstaltungsort in Form einer Schulnummer. Die ursprünglichen $N=1239$ Einträge wurden ebenso in Anlehnung von Richter et al. (2020) bereinigt. Für die Analysen werden Weiterbildungs- und Qualifizierungsangebote sowie Beratungsveranstaltungen und Arbeitskreistreffen ausgeschlossen. Ausgefallene Fortbildungen $(n=43)$ sind im Datensatz erhalten geblieben, da das Forschungsinteresse der Analyse aller angebotenen Veranstaltungen galt. Der bereinigte Datensatz umfasst $N=634$ schulinterne Fortbildungen.

Die internen und externen Fortbildungen wurden anhand ihrer Veranstaltungstitel kategorisiert. Dazu wurden vorab in Anlehnung an die Kategorisierungsvorschläge des IQB-Bildungstrends 2018 (Richter et al. 2019) und der Kategorisierung von Pasternack et al. (2017) sechs thematische Kategorien gebildet (Beschreibung der Kategorien unter 5). Jede interne und externe Fortbildungsveranstaltung wurde einer dieser Kategorien zugeordnet.

Zusätzlich zu den Daten der Fortbildungsdatenbank liegen Daten zu den Merkmalen der Schulen im Land Brandenburg vor. Hierin enthalten sind für alle Schulen Informationen zu Merkmalen der Schülerinnen und Schüler (z.B. Anteil der Schülerinnen und Schüler mit sonderpädagogischen Förderbedarf), des Lehrkräftekollegiums (z.B. Anteil der Lehrkräfte im Seiteneinstieg) sowie zu verschiedenen schulischen Leistungsdaten (z. B. VERA-Vergleichsarbeiten). Der Datensatz umfasst alle $N=658$ staatlichen, allgemeinbildenden Schulen des Landes aus dem Schuljahr 2016/17. Mittels der Schulnummer konnten die schulinternen Fortbildungen den jeweiligen Schulen zugeordnet werden, sodass ein Datensatz vorliegt, der die jewei- 
lige Schule mit ihren Merkmalen und den dazugehörigen SchiLF für die Schuljahre 2016/17 und 2017/18 enthält.

\section{Beschreibung der Variablen}

Entsprechend der vorangestellten Fragestellung werden die Variablen zur Anzahl der SchiLF, zur Beschreibung des Themas der Veranstaltungen sowie vier Variablen zur Beschreibung schulischer Merkmale untersucht (Tab. 1).

\section{Datenanalyse}

Um die Forschungsfrage $1 \mathrm{zu}$ bearbeiten, die sich auf den Umfang der angebotenen SchiLF bezieht, wurde die Anzahl der SchilF pro Schule bestimmt. Für die Analyse der Häufigkeit angebotener Themen im Rahmen von internen und externen Fortbildungsangeboten aus dem Schuljahr 2016/17 (Forschungsfrage 2) wurden die in den Datensätzen aufgelisteten Fortbildungen anhand ihres Veranstaltungstitels einem von sechs Themenschwerpunkten zugeordnet: Curricula, Unterrichtsentwicklung, lehrkräftebezogene Professionalisierung, Inklusion/Integration von Schülerinnen und Schülern mit sonderpädagogischen Förderbedarf, Vermittlung fachlicher Themen im Unterricht (Fachdidaktik) und Schulentwicklung und -organisation. Eine Übersicht der Themen mit einer Beschreibung der Kategorien und Beispielfortbildungen befindet sich im Anhang (Tab. A1). Um eine objektive Zuordnung der Fortbildungen zu Themenschwerpunkten zu erzielen, wurde die Codierung von zwei Ratern unabhängig voneinander für alle Fortbildungen durchgeführt. Die Zuordnung der Fortbildungen erfolgte durch den Erstautor und eine studentische Hilfskraft. Zunächst wurde die erste Hälfte der schulinternen Fortbildungsveranstaltungen unabhängig von beiden Ratern codiert, dann fand ein Austausch zwischen den Ratern statt und abweichende Fälle wurden diskutiert. Anschließend codierten die Rater die zweite Hälfte der schulinternen Fortbildungsveranstaltungen. Mit dem Datensatz der externen Fortbildungen wurde in gleicher Weise verfahren, sodass bei der Hälfte der Veranstaltungen ein Austausch zwischen den Ratern stattfand. Die Beurteilerübereinstimmung kann bei dem schulinternen $(\kappa=0,80)$ als auch dem schulexternen Fortbildungsangebotsdatensatz $(\kappa=0,74)$ als gut eingestuft werden (Wirtz und Kutschmann 2007). Abschließend wurden Abweichungen zwischen den Ratern diskutiert und in einem Konsensverfahren vereinheitlicht, sodass eine gemeinsame Interpretation beider Rater vorlag. Mit Ausnahme einer schulinternen Fortbildung konnten alle Veranstaltungen anhand ihres Titels eindeutig einem Thema zugeordnet werden. Diese Veranstaltung wird von den Analysen ausgeschlossen. Nachfolgend wurde die jeweilige Anzahl der internen und externen Fortbildungsveranstaltungen zu einem der sechs Themenschwerpunkte ermittelt.

Um signifikante Unterschiede zwischen der Fortbildungsanzahl zu einem Themenschwerpunkt im schulinternen oder -externen Fortbildungsformat festzustellen, wurde ein Chi-Quadrat-Test durchgeführt. Dieser zielt darauf ab, beobachtete und erwartete Häufigkeiten miteinander zu vergleichen und damit die Unabhängigkeit 
Tab. 1 Beschreibung der verwendeten Variablen

\begin{tabular}{|c|c|c|c|c|c|c|}
\hline \multirow[t]{2}{*}{ Variablenbezeichnung } & \multirow[t]{2}{*}{ Variablenbeschreibung } & \multirow[t]{2}{*}{$N$} & \multirow[t]{2}{*}{$M$} & \multirow[t]{2}{*}{$S D$} & \multicolumn{2}{|c|}{ Range } \\
\hline & & & & & Min & Max \\
\hline $\begin{array}{l}\text { Anzahl schulinter- } \\
\text { ner Fortbildungen } \\
\text { pro Schule }\end{array}$ & $\begin{array}{l}\text { Anzahl der schulin- } \\
\text { ternen Fortbildungs- } \\
\text { veranstaltungen pro } \\
\text { Schule innerhalb } \\
\text { der zwei Schul- } \\
\text { jahre } 2016 / 17 \text { und } \\
2017 / 18\end{array}$ & 634 & 0,97 & 1,77 & 0 & 22 \\
\hline \multicolumn{7}{|c|}{ Merkmale der Fortbildungen $^{a}$} \\
\hline \multicolumn{7}{|c|}{ Themenschwerpunkte der Fortbildungen } \\
\hline Curricula & $\begin{array}{l}\text { Fortbildungen, die } \\
\text { sich im Titel auf den } \\
\text { Rahmenlehrplan } \\
\text { oder das (schulin- } \\
\text { terne) Curriculum } \\
\text { beziehen }\end{array}$ & 188 & 0,29 & 0,74 & 0 & 6 \\
\hline $\begin{array}{l}\text { Unterrichts- } \\
\text { entwicklung }\end{array}$ & $\begin{array}{l}\text { Fortbildungen, die } \\
\text { sich auf die Gestal- } \\
\text { tung von Unterricht } \\
\text { mit dem Ziel der } \\
\text { Qualitätssteigerung } \\
\text { beziehen }\end{array}$ & 163 & 0,25 & 0,96 & 0 & 19 \\
\hline $\begin{array}{l}\text { Lehrkräftebezogene } \\
\text { Professionalisie- } \\
\text { rung }\end{array}$ & $\begin{array}{l}\text { Fortbildungen, die } \\
\text { sich auf das Lernen } \\
\text { von Lehrkräften zu } \\
\text { Themen außerhalb } \\
\text { des Unterrichts } \\
\text { beziehen }\end{array}$ & 119 & 0,18 & 0,88 & 0 & 18 \\
\hline Fachdidaktik & $\begin{array}{l}\text { Fortbildungen, die } \\
\text { sich im Titel auf } \\
\text { die Didaktik eines } \\
\text { bestimmten Faches } \\
\text { beziehen }\end{array}$ & 32 & 0,05 & 0,24 & 0 & 2 \\
\hline $\begin{array}{l}\text { Inklusion/ } \\
\text { Integration von } \\
\text { SuS mit sonder- } \\
\text { pädagogischen } \\
\text { Förderbedarf }\end{array}$ & $\begin{array}{l}\text { Fortbildungen, die } \\
\text { sich auf den Um- } \\
\text { gang mit SuS mit } \\
\text { einem sonderpäd- } \\
\text { agogischen Förder- } \\
\text { schwerpunkt und/ } \\
\text { oder auf Gemein- } \\
\text { samen Unterricht } \\
\text { beziehen }\end{array}$ & 99 & 0,15 & 0,54 & 0 & 6 \\
\hline $\begin{array}{l}\text { Schulentwicklung } \\
\text { und -organisation }\end{array}$ & $\begin{array}{l}\text { Fortbildungen, die } \\
\text { sich auf schulweite } \\
\text { Entwicklungsziele } \\
\text { und/oder das Schul- } \\
\text { programm beziehen }\end{array}$ & 33 & 0,05 & 0,27 & 0 & 3 \\
\hline
\end{tabular}


Tab. 1 (Fortsetzung)

\begin{tabular}{|c|c|}
\hline \multicolumn{2}{|c|}{ Merkmale der Schulen } \\
\hline $\begin{array}{l}\text { Anzahl der SuS } \\
\text { im Schuljahr } 2016\end{array}$ & $\begin{array}{l}\text { Diese Variable bezieht sich auf die Gesamtanzahl der SuS einer Schule und diente } \\
\text { als Indikator für die Größe einer Schule }\end{array}$ \\
\hline $\begin{array}{l}\text { Anteil der SuS } \\
\text { mit sonder- } \\
\text { pädagogischen } \\
\text { Förderbedarf }\end{array}$ & $\begin{array}{l}\text { Diese Variable umfasst den prozentualen Anteil der SuS einer Schule, welche } \\
\text { einen festgestellten Bedarf an sonderpädagogischer Unterstützung in den Berei- } \\
\text { chen Lernen, emotionale und soziale Entwicklung oder Sprache aufweisen }\end{array}$ \\
\hline $\begin{array}{l}\text { Anteil der SuS, } \\
\text { die den Min- } \\
\text { deststandard in } \\
\text { den VERA-Ver- } \\
\text { gleichsarbeiten } \\
\text { erreicht haben }{ }^{\text {b }}\end{array}$ & $\begin{array}{l}\text { Diese Variable repräsentiert den gemittelten prozentualen Anteil der SuS einer } \\
\text { Schule, die den Mindeststandard im Rahmen von VERA-3 in Deutsch und Mathe- } \\
\text { matik beziehungsweise VERA- } 8 \text { für die Sekundarstufe I in Deutsch, Mathematik } \\
\text { und Englisch erreicht haben }\end{array}$ \\
\hline $\begin{array}{l}\text { Anteil der Lehr- } \\
\text { kräfte im Sei- } \\
\text { teneinstieg im } \\
\text { Kollegium }^{\text {b }}\end{array}$ & $\begin{array}{l}\text { Diese Variable gibt an, zu welchem prozentualen Anteil Lehrkräfte im Kollegium } \\
\text { einer Schule vertreten sind, die kein traditionelles Lehramtsstudium absolviert } \\
\text { haben und über einen alternativen Zugang in den Schuldienst eingemündet sind }\end{array}$ \\
\hline
\end{tabular}

Berichtet werden die Anzahl $(N)$, Mittelwerte $(M)$ und Standardabweichung $(S D)$

SuS Schülerinnen und Schüler

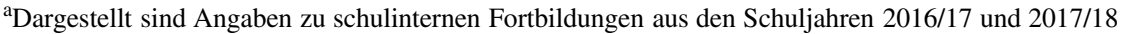

${ }^{b}$ Schulische Merkmale können Werte im Bereich zwischen 0 und 100\% annehmen. Höhere Prozentwerte stellen einen höheren Anteil an der jeweiligen Gesamtheit der Einzelschule dar

der Variablen voneinander zu überprüfen. In den Feldern der erzeugten Kreuztabelle werden neben der beobachteten und erwarteten Anzahl der Veranstaltungen die standardisierten Residuen ausgegeben, die als z-Werte interpretiert werden können. Liegt der z-Wert hierfür über dem Grenzwert von |1,96|, zeigt er für die jeweilige Zelle an, ob mehr oder weniger Fortbildungen als erwartet angeboten wurden (Bühl 2006). Hierzu wird Cramers $V$ als Maß für die Effektstärke des Tests berichtet. Dieser kann Werte zwischen 0 und 1 annehmen. Höhere Werte deuten dabei auf einen stärkeren Zusammenhang hin. Die Grenzen liegen bei Cramers $V=0,10$ kleiner Effekt, Cramers $V=0,30$ mittlerer Effekt und Cramers $V=0,50$ für einen großen Effekt (Cohen 1988). Für den paarweisen Vergleich der Spaltenanteile wurde die Bonferroni-Korrektur angewendet, die das beobachtete Signifikanzniveau für Mehrfachvergleiche anpasst, um eine Alpha-Fehler-Kumulierung zu vermeiden.

Für die Bearbeitung der Forschungsfrage 3 (Zusammenhang zwischen Schulmerkmalen und den Themen von SchiLF) wurde zu jedem Themenschwerpunkt eine multiple Regressionsanalyse durchgeführt, um die Effekte einzelner schulischer Merkmale auf das gewählte Thema der SchiLF unter Kontrolle der Kovariaten zu überprüfen. Insgesamt wurde sechs Modelle berechnet. Hierfür dienten die folgenden schulischen Merkmale als unabhängige Variablen: Anzahl der Schülerinnen und Schüler in der Schule, der Anteil der Schülerinnen und Schüler mit sonderpädagogischen Förderbedarf, der Anteil der Schülerinnen und Schüler, die den Mindeststandard in den VERA-Vergleichsarbeiten erreicht haben und Anteil an Lehrkräften im Seiteneinstieg im Kollegium. Die jeweilige Anzahl der SchiLF-Veranstaltungen zu einem der sechs Themenschwerpunkte (kontinuierliche Variable) stellte die abhängige Variable dar. Fehlende Werte lagen in den analysierten Daten nicht vor. Alle Analysen wurden mit dem Programm IBM SPSS Statistics 26 durchgeführt. 


\section{Ergebnisse}

Bezüglich des Umfangs des SchiLF-Angebots zeigt sich, dass in den Schuljahren 2016/17 und 2017/18 insgesamt 634 Fortbildungen an 285 allgemeinbildenden Schulen angeboten wurden. Im Schuljahr 2016/17 wurden etwas mehr als die Hälfte der SchiLF angeboten (64,8\% der Veranstaltungen in 2016/17). Von insgesamt 658 allgemeinbildenden Schulen boten demnach 373 Schulen $(56,7 \%)$ im Untersuchungszeitraum keine SchiLF an (Abb. 2). Weitere 31,5\% der Schulen boten ein bis zwei Fortbildungen an, 5,0\% boten drei Fortbildungen an und 6,9\% der Schulen führten vier oder mehr Fortbildungen durch. Somit boten die Schulen über zwei Schuljahre hinweg im Durchschnitt 0,97 Veranstaltungen (bei insgesamt 658 Schulen; $S D=1,77$ ) an.

Der Vergleich der Häufigkeiten schulinterner und externer Fortbildungsthemen aus dem Schuljahr 2016/17 zeigt - wie in Abb. 3 verdeutlicht -, dass am häufigsten SchiLF zu Themen der Curricula (39\% aller Fortbildungen), Unterrichtsentwicklung (ca. 23\%) und lehrkräftebezogene Professionalisierung (ca. 15\%) angeboten werden. SchiLF zum Thema Inklusion/Integration nimmt einen Anteil von ca. $12 \%$ ein. Eher selten werden SchiLF zu den Themen Schulentwicklung und-organisation (ca. 6\%) und Fachdidaktik (ca. 5\%) angeboten.

Zur Einordnung des Umfangs der im Rahmen von SchiLF angebotenen Themen werden in Abb. 3 auch die Angebote externer Veranstaltungen dargestellt. Bei den externen Fortbildungsangeboten werden am häufigsten Veranstaltungen zum Themenschwerpunkt Curricula (ca. $44 \%$ aller externen Fortbildungen) angeboten. Es folgen Veranstaltungen zum Thema Fachdidaktik (ca. $25 \%$ ), Unterrichtsentwicklung (ca. 13\%) und Inklusion/Integration (ca. 9\%). Fortbildungen zur lehrkräftebezoge-

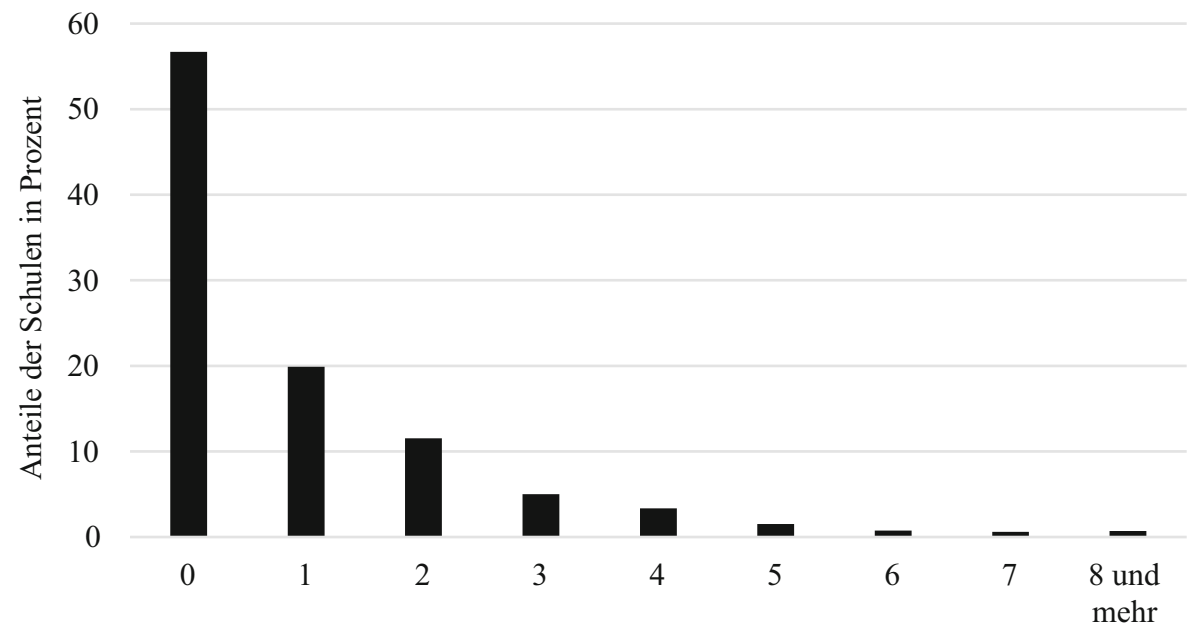

SchiLF-Angebot pro Schule im Zeitraum der Schuljahre 2016/17-2017/18

Abb. 2 Prozentuale Anteile der Schulen, die in den Schuljahren 2016/17 und 2017/18 in einem bestimmten Umfang SchiLF angeboten haben 
Curricula (Rahmenlehrplan, Lehrpläne)

\author{
Unterrichtsentwicklung \\ Lehrkräftebezogene \\ Professionalisierung \\ Fachdidaktik \\ Inklusion/ Integrtaion von SuS mit \\ sonderpädagogischen \\ Förderschwerpunkt
}

Schulentwicklung und -organisation

- Schulexterne Fortbildungen

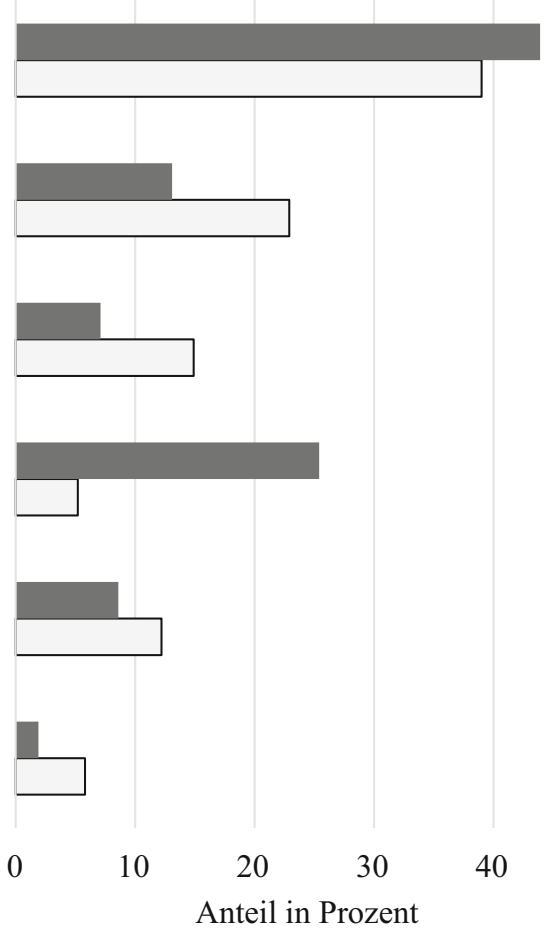

$\square$ Schulinterne Fortbildungen

Abb. 3 Prozentuale Anteile der internen und externen Fortbildungsangebote nach Themenschwerpunkten. (SuS Schülerinnen und Schüler)

nen Professionalisierung nehmen einen Anteil von ca. $7 \%$ ein. SchiLF zur Schulentwicklung und -organisation ist im externen Fortbildungsangebot ebenfalls eher selten vertreten (ca. 2\%).

Die Ergebnisse des Chi-Quadrat-Tests, dargestellt in Tab. 2, verweisen zunächst auf einen signifikanten Zusammenhang zwischen Thema und Format der Fortbildung mit einer mittleren Effektstärke $\left(\chi^{2}(5)=104,78 ; p<0,001\right.$; Cramers $\left.V=0,29\right)$. Der Vergleich der Spaltenanteile zeigt, dass signifikante Unterschiede zwischen den Angebotsformaten bei den Themen Fachdidaktik, lehrkräftebezogene Professionalisierung, Unterrichtsentwicklung und Schulentwicklung und-organisation bestehen. Fortbildungen zum Thema Fachdidaktik werden häufiger als erwartet extern angeboten $(z=8,2)$. Fortbildungen zu den Themen lehrkräftebezogene Professionalisierung $(z=4,4)$, Unterrichtsentwicklung $(z=4,4)$ und Schulentwicklung und -organisation $(z=3,7)$ werden hingegen häufiger als erwartet schulintern angeboten. Zusammengefasst wird deutlich, dass sich die Häufigkeit der Fortbildungsthemen in Abhängigkeit vom Fortbildungsformat unterscheidet.

Die Ergebnisse der Regressionsanalysen (dargestellt in Tab. 3 und 4), ergeben signifikante Zusammenhänge zwischen schulischen Merkmalen und einzelnen SchiLFThemen für die Modelle 3 und 5. Für das Modell 3 zeigt sich zunächst ein sig- 
Tab. 2 Kreuztabelle zur Anzahl der Fortbildungen pro Thema und Fortbildungsformat mit Residuen

\begin{tabular}{|c|c|c|c|}
\hline \multirow{2}{*}{$\begin{array}{l}\text { Thema der } \\
\text { Fortbildung }\end{array}$} & & \multicolumn{2}{|l|}{ Fortbildungsformat } \\
\hline & & $\begin{array}{l}\text { Schulinterne Fort- } \\
\text { bildungen }\end{array}$ & $\begin{array}{l}\text { Schulexterne Fort- } \\
\text { bildungen }\end{array}$ \\
\hline \multirow[t]{3}{*}{$\overline{\text { Curricula }}$} & Beobachtete Häufigkeit & 141 & 409 \\
\hline & Erwartete Anzahl & 153,9 & 396,1 \\
\hline & $\begin{array}{l}\text { Korrigierte standardisierte } \\
\text { Residuen }\end{array}$ & $-1,6 \mathrm{a}$ & $1,6 \mathrm{a}$ \\
\hline \multirow[t]{3}{*}{ Unterrichtsentwicklung } & Beobachtete Häufigkeit & 83 & 122 \\
\hline & Erwartet Anzahl & 57,3 & 147,7 \\
\hline & $\begin{array}{l}\text { Korrigierte standardisierte } \\
\text { Residuen }\end{array}$ & $4,4 a$ & $-4,4 b$ \\
\hline \multirow{3}{*}{$\begin{array}{l}\text { Lehrkräftebezogene } \\
\text { Professionalisierung }\end{array}$} & Beobachtete Häufigkeit & 54 & 66 \\
\hline & Erwartete Anzahl & 33,6 & 86,4 \\
\hline & $\begin{array}{l}\text { Korrigierte standardisierte } \\
\text { Residuen }\end{array}$ & $4,4_{a}$ & $-4,4_{b}$ \\
\hline \multirow[t]{3}{*}{ Fachdidaktik } & Beobachtete Häufigkeit & 19 & 237 \\
\hline & Erwartete Anzahl & 71,6 & 184,4 \\
\hline & $\begin{array}{l}\text { Korrigierte standardisierte } \\
\text { Residuen }\end{array}$ & $-8,2 \mathrm{a}$ & $8,2_{b}$ \\
\hline \multirow{3}{*}{$\begin{array}{l}\text { Inklusion/Integration } \\
\text { von SuS mit } \\
\text { sonderpädagogischen } \\
\text { Förderbedarf }\end{array}$} & Beobachtete Häufigkeit & 44 & 80 \\
\hline & Erwartete Anzahl & 34,7 & 89,3 \\
\hline & $\begin{array}{l}\text { Korrigierte standardisierte } \\
\text { Residuen }\end{array}$ & $2,0 \mathrm{a}$ & $-2,0 \mathrm{a}$ \\
\hline \multirow{3}{*}{$\begin{array}{l}\text { Schulentwicklung und } \\
\text {-organisation }\end{array}$} & Beobachtete Häufigkeit & 21 & 18 \\
\hline & Erwartete Anzahl & 10,9 & 28,1 \\
\hline & $\begin{array}{l}\text { Korrigierte standardisierte } \\
\text { Residuen }\end{array}$ & $3,7_{\mathbf{a}}$ & $-3,7_{b}$ \\
\hline
\end{tabular}

Dargestellt sind beobachtete und erwartete Häufigkeiten der Fortbildungen pro Thema und Fortbildungsformat. Fettgedruckte Koeffizienten liegen über dem Grenzwert von $z=|1,96|$ und markieren eine statistisch signifikante Abweichung der beobachteten von der erwarteten Häufigkeit. Für den paarweisen Vergleich der Spaltenanteile wurde eine Bonferroni-Korrektur genutzt, die das beobachtete Signifikanzniveau für Mehrfachvergleiche anpasst. Signifikante Unterschiede zwischen den Spaltenpaaren werden durch unterschiedliche tiefgestellte Buchstaben markiert

SuS Schülerinnen und Schüler

nifikanter Zusammenhang zwischen dem Anteil der Schülerinnen und Schülern, die den Mindeststandard in den VERA-Vergleichsarbeiten erreicht haben, und der Fortbildungsanzahl zum Thema lehrkräftebezogene Professionalisierung. Bei einem zunehmenden Anteil der Schülerinnen und Schülern, die den Mindeststandard in den VERA-Vergleichsarbeiten erreicht haben, werden weniger SchiLF zum Thema lehrkräftebezogene Professionalisierung angeboten. Außerdem ergab sich in Modell 3 ein signifikanter Zusammenhang zwischen dem Anteil an Lehrkräften im Seiteneinstieg und der Fortbildungsanzahl. Mit einem steigenden Anteil an Lehrkräften im Seiteneinstieg im Kollegium bietet die Schule mehr SchiLF zum Thema lehrkräftebezogene Professionalisierung an.

Die Ergebnisse für das Modell 5 zeigen, dass ein signifikanter Zusammenhang zwischen dem Anteil der Schülerinnen und Schülern mit sonderpädagogischen För- 


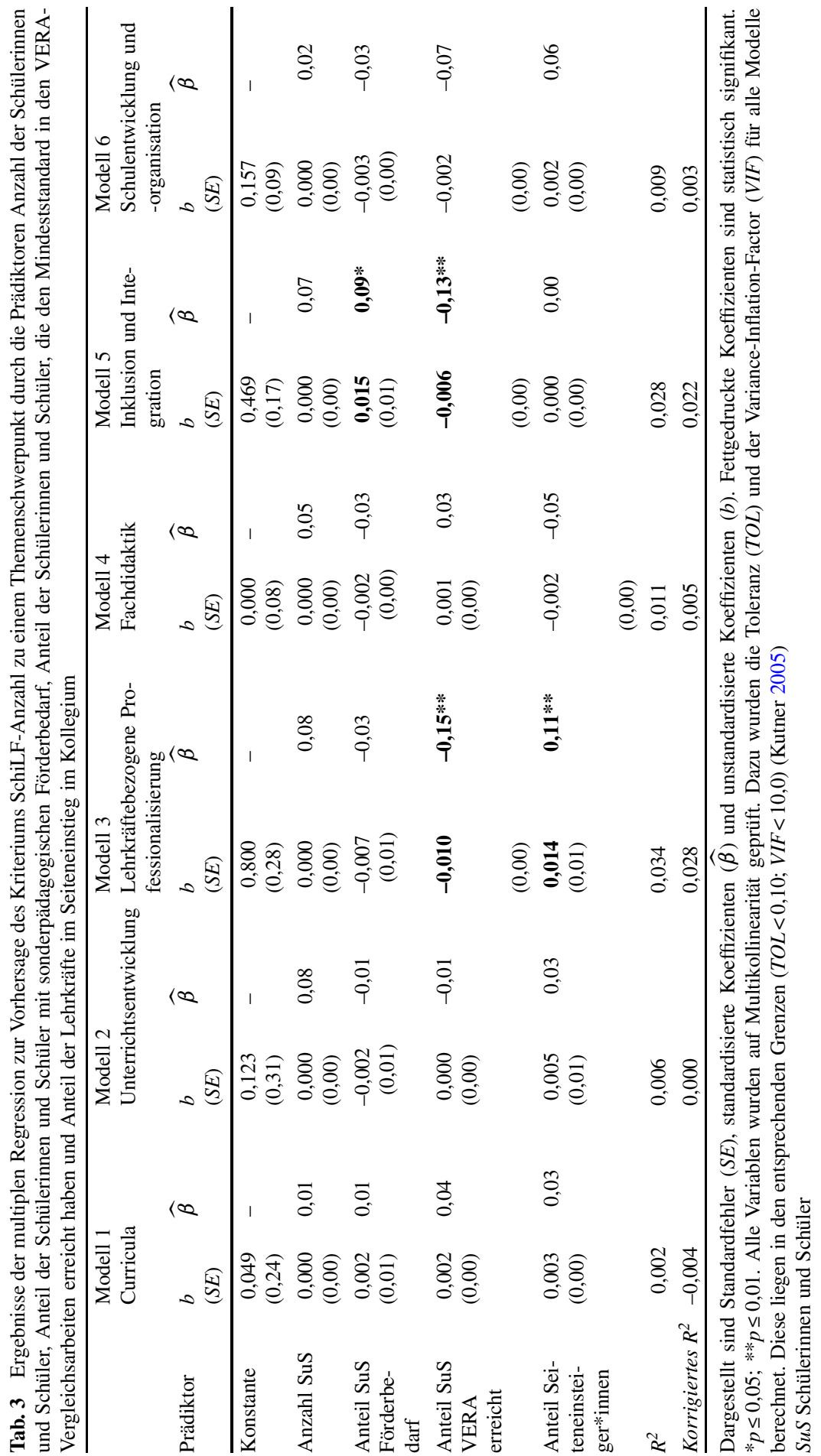


Tab. 4 Ergebnisse der Varianzanalysen zur multiplen Regression

\begin{tabular}{llcc}
\hline Modell & $F$ & $d f$ & $p$ \\
\hline Curricula & 0,32 & 4 & 0,87 \\
Unterrichtsentwicklung & 0,93 & 4 & 0,42 \\
Lehrkräftebezogene Professionalisierung & 5,65 & 4 & $<0,001$ \\
Fachdidaktik & 1,84 & 4 & 0,12 \\
Inklusion und Integration & 4,62 & 4 & $<0,01$ \\
Schulentwicklung und -organisation & 1,51 & 4 & 0,20 \\
\hline
\end{tabular}

Berichtet werden die Ergebnisse der ANOVA zur Regressionsanalyse. Dargestellt sind $F$-Wert $(F)$, Freiheitsgrade $(d f)$ und Signifikanzniveau $(p)$

derbedarf und der Fortbildungsanzahl zum Thema Inklusion/Integration besteht. Das bedeutet, dass Schulen bei einem zunehmenden Anteil an Schülerinnen und Schülern mit sonderpädagogischen Förderbedarf mehr Veranstaltungen zum Thema Inklusion/ Integration anbieten. Weiterhin kann in Bezug zu Modell 5 ein signifikanter Zusammenhang zwischen dem Anteil an Schülerinnen und Schülern, die den Mindeststandard in den VERA-Vergleichsarbeiten erreicht haben und der Fortbildungsanzahl zum Thema Inklusion/Integration aufgezeigt werden. Bei einem zunehmenden Anteil an Schülerinnen und Schülern, die den Mindeststandards erreichen, bietet die Schule weniger SchiLF zum Thema Inklusion/Integration an. Insgesamt ist bei der Interpretation der Ergebnisse zu berücksichtigen, dass durch die Modelle 3 und 5 jeweils lediglich $3 \%$ der Varianz erklärt werden können. Die niedrigen Werte des korrigierten $R^{2}$ (Modell 3: $R^{2}{ }_{k o r r}=0,03$; Modell 5: $R^{2}{ }_{\text {korr }}=0,02$ ) deuten darauf hin, dass das gewählte Modell nur eingeschränkt für die Analyse der vorliegenden Daten geeignet ist. Weitere signifikante Zusammenhänge können in den übrigen vier Modellen nicht festgestellt werden.

\section{Diskussion}

Die vorliegende Studie untersucht, in welchem Umfang allgemeinbildende Schulen SchiLF anbieten, welche thematischen Schwerpunkte dabei im Vordergrund stehen und inwiefern hierbei ein Unterschied zwischen schulinternem und schulexternem Angebot vorliegt. Des Weiteren greifen wir die Frage auf, ob Zusammenhänge zwischen den Merkmalen einer Schule und der Anzahl an SchiLF zu einem bestimmten Themenschwerpunkt bestehen.

Hinsichtlich der Forschungsfrage 1, in welchem Umfang SchiLF angeboten werden, kann gezeigt werden, dass die knappe Mehrheit der Schulen (ca. 57\%) ihren Lehrkräften im Untersuchungszeitraum von zwei Schuljahren keine SchiLF durch öffentliche Anbieter unterbreitete. Es gibt nur sehr wenige Schulen, die mehr als eine Fortbildung durchführen. Im Durchschnitt bietet eine Schule in zwei Schuljahren nur eine SchiLF an. Bereits in früheren Untersuchungen in Brandenburg und Thüringen wurde deutlich, dass nicht von allen Schulen schulinterne Fortbildungsangebote der öffentlicher Träger genutzt werden (DVLfB 2018). Eine mögliche Erklärung für diesen Befund könnte darin liegen, dass Schulen neben staatlichen Angeboten auch von Fortbildungsangeboten nichtstaatlicher Anbieter (z.B. Verlage) Gebrauch ma- 
chen und diese deshalb nicht im vorliegenden Datensatz verzeichnet sind. Darüber hinaus verweisen Studienergebnisse auf eine hohe Arbeitsbelastung von Schulleitungen hin (z. B. Brauckmann und Schwarz 2015). Daher wäre es denkbar, dass ihnen wenig Zeit für die Planung und Durchführung von Personalentwicklungsmaßnahmen bleibt, da sie aufgrund vielfältiger administrativer Aufgaben im Schulalltag und eigener Unterrichtsverpflichtung stark belastet sind (Meyer et al. 2019). In Brandenburg sind dies laut der Verwaltungsvorschriften über Anrechnungsstunden für Lehrkräfte (VV-Anrechnungsstunden $§ 2,2008$ ) in Abhängigkeit von der Schulart und Schulgröße maximal zwischen 13 und 15 Unterrichtsstunden pro Woche. Appius et al. (2012) berichten ebenfalls, dass sich Schulleitungen zwar der Wichtigkeit von Personalentwicklung bewusst sind, fehlende zeitliche und finanzieller Ressourcen sowie ein umfangreiches Aufgabenspektrum sie jedoch bei der Umsetzung zeitintensiver Personalentwicklungsmaßnahmen einschränken.

Forschungsfrage 2 untersucht die Häufigkeit der Themen, die im Rahmen von SchiLF angeboten werden und geht auf Unterschiede zum externen Fortbildungsangebot in diesen Häufigkeiten ein. Die Analysen ergeben zunächst einen Schwerpunkt beim Thema Curricula sowohl im internen als auch im externen Fortbildungsangebot. Dieses Ergebnis lässt sich damit erklären, dass in Brandenburg ab dem Schuljahr 2017/18 für die Jahrgangsstufen 1 bis 10 ein neuer Rahmenlehrplan galt, welcher von den Schulen in das schulinterne Curriculum integriert werden musste (Missal 2019). Daran wird deutlich, dass administrative Reformbestrebungen schulische Innovationsprozesse binden. Die Analysen zeigen weiterhin, dass die Themenschwerpunkte lehrkräftebezogene Professionalisierung, Unterrichtsentwicklung und Schulentwicklung und -organisation häufiger bei SchiLF-Veranstaltungen auftauchen als bei externen Fortbildungsangeboten. Veranstaltungen mit dem Thema Fachdidaktik sind häufiger bei externen Fortbildungsangeboten vertreten. Damit deuten die Ergebnisse in dieselbe Richtung wie die Befunde von Pasternack et al. (2017). Die Autorinnen und Autoren stellen fest, dass im Rahmen von SchiLF weniger fachdidaktische Inhalte präsent sind, da sich SchiLF in der Regel an das gesamte Kollegium der Schule richten und somit eher Themen beinhalten, die für Lehrkräfte aller Fachrichtungen relevant sind. Darüber hinaus kann anhand der durchgeführten Analysen gezeigt werden, dass die Themen lehrkräftebezogenen Professionalisierung, Unterrichtsentwicklung und Schulentwicklung und -organisation für Lehrkräftekollegien von besonderem Interesse sind.

Forschungsfrage 3 bezog sich auf die Untersuchung des Zusammenhangs zwischen den Merkmalen einer Schule und den gewählten Themen von SchiLF. Die Ergebnisse der Regressionsanalysen zeigen, dass in Teilen Zusammenhänge zwischen Schulmerkmalen und der Nutzung ausgewählter SchiLF-Themen bestehen. Zum einen kann gezeigt werden, dass mit zunehmendem Anteil an Schülerinnen und Schülern mit festgestelltem Bedarf an sonderpädagogischer Unterstützung mehr Fortbildungen zum Themenschwerpunkt Inklusion/Integration angeboten werden. Das Ergebnis deutet zunächst darauf hin, dass sich Schulen mit der Gestaltung inklusiven Unterrichts auseinandersetzen. Darüber hinaus könnte der Befund damit erklärt werden, dass die Schulen, die nach dem 2015 beschlossenen Konzept der Brandenburger Landesregierung eine „Schule für gemeinsames Lernen“ werden wollen, dazu einen verbindlichen Anteil an SchiLF zu diesem Thema absolvieren 
müssen (Landesregierung Brandenburg 2015). Insofern ist anzunehmen, dass ein Teil der Schulen das SchiLF-Thema Inklusion/Integration nicht gänzlich frei auswählte, sondern einer Vorgabe im Rahmen dieser Maßnahme folgte.

Weiterhin zeigen die Analysen zur Forschungsfrage 3, dass bei einem höheren Anteil an Schülerinnen und Schülern, die den Mindeststandard in den Vergleichsarbeiten erreicht haben, weniger SchiLF zum Thema Inklusion/Integration und zu lehrkräftebezogene Professionalisierung gewählt werden. Eventuell verweisen die Ergebnisse darauf, dass Schulen aufgrund ihrer Rückmeldungen aus den Vergleichsarbeiten andere Fortbildungsthemen in den Fokus rücken und die Rückmeldungen eher als Grundlage für die Unterrichtsentwicklung nutzen (Wurster et al. 2017) hierzu sind weitere Untersuchungen notwendig.

Schließlich kann gezeigt werden, dass bei einem höheren Anteil an Lehrkräften im Seiteneinstieg im Kollegium mehr SchiLF zur lehrkräftebezogenen Professionalisierung gewählt werden. Da Lehrkräften im Seiteneinstieg nicht im gleichen Umfang wie traditionell ausgebildete Lehrkräfte auf den Schuldienst vorbereitet sind (Lucksnat et al. 2020), kann dieser Befund in der Art gedeutet werden, dass SchiLF eine Form der Unterstützung beim Berufseinstieg für Lehrkräfte im Seiteneinstieg darstellen.

Insgesamt können für alle untersuchten Modelle zum Zusammenhang zwischen schulischen Merkmalen und SchiLF-Themen jedoch nur gering ausgeprägte Effektstärken festgestellt werden. Das bedeutet, dass die untersuchten schulischen Merkmale nur zu einem kleinen Teil mit den Themen der SchiLF in Verbindung stehen. Fischer (2017) berichtet hierzu in ihrer qualitativen Studie zu internen Fortbildungen an Schweizer Berufsfachschulen, dass an einigen Schulen die Auswahl der Inhalte kurzfristig und unsystematisch erfolgte. Im Hinblick auf die vorliegenden Ergebnisse könnte dies ein Hinweis darauf sein, dass die Auswahl eines SchiLF-Themas zum Teil nicht systematisch mit den individuellen Rahmenbedingungen der Schulen korrespondiert.

Damit leistet diese Arbeit einen Beitrag für die Bildungsforschung, da die Ergebnisse zum Zusammenhang zwischen Schulmerkmalen und Fortbildungsthemen verdeutlichen, dass SchiLF eine wichtige Funktion für die Entwicklung der Einzelschule innehaben, jedoch machen scheinbar nicht alle Schulen vom staatlichen Angebot umfänglich Gebrauch. Die Ergebnisse zeigen weiterhin, dass in zukünftigen Forschungsarbeiten eine differenzierte Betrachtung von schulinternen und -externen Fortbildungen sinnvoll ist. Da die Angebotsformate jeweils unterschiedliche Ziele und Themenschwerpunkte adressieren, könnte eine differenzierte Betrachtung Aufschluss auf die jeweiligen Fortbildungsbedarfe auf der Ebene der Einzelschule beziehungsweise der Lehrperson geben.

Anhand der Ergebnisse ergeben sich zudem Implikationen für die schulische Praxis. Da nicht alle Schulen von SchiLF durch öffentliche Anbieter Gebrauch machen, sollte darüber nachgedacht werden, wie Schulleitungen bei der datenbasierten Bedarfsanalyse und Planung sowie bei der Durchführung von SchiLF unterstützt und im Hinblick auf ihr hohes Arbeitspensum entlastet werden könnten. Eine Möglichkeit bestände darin, Fortbildungsbeauftragte aus dem Lehrkräftekollegium stärker bei der Planung und Durchführung von Fortbildungen mit einzubeziehen. Darüber hinaus könnte die Verbindlichkeit durch regelmäßige und feste Zeitfenster (Richter 
et al. 2020) sowie eine schriftliche Planung erhöht werden (Appius et al. 2012). Ob eine strengere gesetzliche Verpflichtung zur Durchführung von schulinternen Fortbildungen eine stärkere Nutzung bewirken könnte, ist jedoch fraglich (Kuschel et al. 2020). Darüber hinaus können die Ergebnisse auch dahingehend gedeutet werden, dass das Fortbildungsangebot in der Wahrnehmung der Schulen keine hinreichende Qualität aufweist. Es wäre z.B. denkbar, dass SchiLF eher von Lehrkräften aus dem Kollegium in Form von Mikrofortbildungen durchgeführt werden (Wöhlbier et al. 2019), da diese im Gegensatz zu externen Referenteninnen und Referenten mit den Rahmenbedingungen der Einzelschule vertraut sind und das Fortbildungsangebot entsprechend passend gestalten können.

$\mathrm{Zu}$ den Limitationen der vorliegenden Arbeit zählt, dass nur Daten eines Bundeslandes ausgewertet wurden und eine Übertragung der Ergebnisse auf andere Bundesländer zu prüfen ist. Darüber hinaus stellen die Ergebnisse zur Forschungsfrage 3 eine Querschnittsanalyse dar. Um Zusammenhänge zwischen Schulmerkmalen und der Nutzung von SchiLF zu überprüfen, ist eine Untersuchung im Längsschnitt über mehrere Schuljahre aussagekräftiger. Mit Hilfe des verwendetet Modells kann ferner nur ein kleiner Teil der Varianz in den Daten aufgeklärt werden kann. Das bedeutet, dass die betrachteten Schulmerkmale nur in einem geringen Maße mit den gewählten SchiLF-Themen zusammenhängen. Somit gibt es noch weitere ungeklärte Faktoren, die für die Themenwahl bedeutsam sind. Unter anderem wäre es möglich, dass die Führungskompetenz der Schulleitung eine entscheidende Rolle spielt. Appius et al. (2012) sowie Steger Vogt und Kabitz (2014) stellen fest, dass die Fähigkeit, Lehrkräfte zu motivieren und klare Zielevereinbarungen zu treffen, für die Umsetzung von Maßnahmen von Bedeutung sind. Schließlich muss darauf hingewiesen werden, dass in der vorliegenden Arbeit nur Angebote der staatlichen Schulämter berücksichtigt werden, die im Datensatz verzeichnet sind.

Dessen ungeachtet stellt die vorliegende Untersuchung erstmalig die Häufigkeit spezifischer SchiLF-Angebote in der Gesamtheit der Schulen eines Bundeslandes dar und untersucht Zusammenhänge zwischen Schulmerkmalen und Themenschwerpunkten. Damit trägt diese Studie dazu bei, anhand von schulspezifischen Merkmalen Fortbildungs- und Unterstützungsbedarfe dieser Schulen aufzuzeigen.

Insgesamt verdeutlichen die Ergebnisse, dass weiterer Forschungsbedarf zu schulinternen Fortbildungen besteht. Zentrale Aspekte stellen die Untersuchung der Qualität schulinterner Fortbildungen sowie deren Effektivität und Nachhaltigkeit dar. Trotz der Bedeutung, die schulinternen Fortbildungen für den Kompetenzerwerb im Kollegium und die Schulentwicklung zugesprochen wird, liegen hierzu derzeitig keinerlei Forschungsergebnisse vor. Aufgrund dieser zunehmenden Bedeutsamkeit von SchiLF für Schulentwicklung (Pasternack et al. 2017) sind weitere Untersuchungen zu SchiLF von großer Relevanz für die aktuelle Bildungsforschung. 
Anhang

Tab. A1 Definition der Themenschwerpunkte mit Beispielfortbildungen

\begin{tabular}{|c|c|c|}
\hline Themenschwerpunkt mit Definition & $\begin{array}{l}\text { Beispiele für interne Fortbil- } \\
\text { dungen }\end{array}$ & $\begin{array}{l}\text { Beispiele für externe Fort- } \\
\text { bildungen }\end{array}$ \\
\hline \multirow{2}{*}{$\begin{array}{l}\text { Curricula (Lehrpläne, RLP): } \\
\text { Fortbildungen, die sich im Titel auf } \\
\text { den Rahmenlehrplan oder das } \\
\text { (schulinterne) Curriculum beziehen }\end{array}$} & Beratung zum SchiC Deutsch & $\begin{array}{l}\text { Arbeit am SchiC - Daten } \\
\text { und Zufall }\end{array}$ \\
\hline & $\begin{array}{l}\text { SchiLF zur Implementierung } \\
\text { RLP }\end{array}$ & $\begin{array}{l}\text { Qualifizierungsmaßnahme } \\
\text { im Rahmen der Implemen- } \\
\text { tierung des RLP }\end{array}$ \\
\hline \multirow{2}{*}{$\begin{array}{l}\text { Unterrichtsentwicklung: } \\
\text { Fortbildungen, die sich auf die } \\
\text { Gestaltung von Unterricht mit dem } \\
\text { Ziel der Qualitätssteigerung } \\
\text { beziehen }\end{array}$} & Energizer und Gruppenbildung & $\begin{array}{l}\text { Die Struktur guten Unter- } \\
\text { richts }\end{array}$ \\
\hline & $\begin{array}{l}\text { Lernmethoden: Visualisierung, } \\
\text { Mindmapping, Lernstrategien }\end{array}$ & Medien für Lehrer \\
\hline \multirow{3}{*}{$\begin{array}{l}\text { Lehrkräftebezogene } \\
\text { Professionalisierung: } \\
\text { Fortbildungen, die sich auf das } \\
\text { Lernen von Lehrkräften zu Themen } \\
\text { außerhalb des Unterrichts beziehen }\end{array}$} & $\begin{array}{l}\text { Konfliktbewältigung Lehrer - } \\
\text { Schulsozialarbeiter }\end{array}$ & $\begin{array}{l}\text { BEP - Mein erster El- } \\
\text { ternabend als Klassenlehr- } \\
\text { kraft }\end{array}$ \\
\hline & $\begin{array}{l}\text { Helicoptereltern fahren auch } \\
\text { bloß Auto }\end{array}$ & Unsicherheitsfaktor Eltern \\
\hline & $\begin{array}{l}\text { Kollegiale Fallberatung als } \\
\text { wirksames Mittel im Problem- } \\
\text { lösungsprozess }\end{array}$ & $\begin{array}{l}\text { Supervision - Umgang mit } \\
\text { schwierigen Beratungssi- } \\
\text { tuationen }\end{array}$ \\
\hline $\begin{array}{l}\text { Vermittlung fachlicher Themen im } \\
\text { Unterricht (Fachdidaktik): } \\
\text { Fortbildungen, die sich im Titel auf } \\
\text { die Didaktik eines bestimmten } \\
\text { Faches beziehen }\end{array}$ & $\begin{array}{l}\text { Einführung in die Verwendung } \\
\text { von Geometriesoftware im } \\
\text { Mathematikunterricht }\end{array}$ & $\begin{array}{l}\text { Epische Literatur des } \\
\text { 20./21. Jahrhundert mit den } \\
\text { Schwerpunkten neue } \\
\text { Medien und } \\
\text { Erzählkonzepte }\end{array}$ \\
\hline \multirow{2}{*}{$\begin{array}{l}\text { Inklusion/Integration von SuS mit } \\
\text { sonderpädagogischen } \\
\text { Förderbedarf: } \\
\text { Fortbildungen, die sich auf den } \\
\text { Umgang mit SuS mit einem } \\
\text { sonderpädagogischen } \\
\text { Förderschwerpunkt und/oder auf } \\
\text { Gemeinsamen Unterricht beziehen }\end{array}$} & $\begin{array}{l}\text { Leistungsbewertung und Nach- } \\
\text { teilsausgleich bei SuS mit und } \\
\text { ohne festgestelltem sonderpäd- } \\
\text { agogischen Förderbedarf }\end{array}$ & $\begin{array}{l}\text { Den schulischen Alltag } \\
\text { für SuS mit Förderbedarf } \\
\text { im autistischen Verhalten } \\
\text { erfolgreich gestalten }\end{array}$ \\
\hline & $\begin{array}{l}\text { Inklusion - Herausforderung } \\
\text { und Chance. Umgang mit Kon- } \\
\text { flikten und Krisensituationen } \\
\text { im Schulalltag }\end{array}$ & $\begin{array}{l}\text { Adaptionen im gemeinsa- } \\
\text { men Unterricht für blinde } \\
\text { SuS }\end{array}$ \\
\hline \multirow{2}{*}{$\begin{array}{l}\text { Schulentwicklung und } \\
\text {-organisation: } \\
\text { Fortbildungen, die sich auf } \\
\text { schulweite Entwicklungsziele und/ } \\
\text { oder das Schulprogramm beziehen }\end{array}$} & $\begin{array}{l}\text { Ganztag im Fokus-Evaluie- } \\
\text { rung und Fortschreibung des } \\
\text { Ganztagskonzeptes }\end{array}$ & $\begin{array}{l}\text { Einsatz der neuen Hard- } \\
\text { ware und der neuen Medien } \\
\text { im Schulnetzwerk }\end{array}$ \\
\hline & $\begin{array}{l}\text { Bewegte Schule als Element } \\
\text { des Schulprogramms }\end{array}$ & $\begin{array}{l}\text { Fachtagung „Qualität und } \\
\text { Perspektiven kultureller } \\
\text { Bildung an Schulen mit } \\
\text { Ganztagsangeboten“ }\end{array}$ \\
\hline
\end{tabular}

SuS Schülerinnen und Schüler, RLP Rahmenlehrplan, SchiC Schulinternes Curriculum, BEP Berufseingangsphase 
Danksagung Wir danken Herrn Michael Wolf vom Ministerium für Bildung, Jugend und Sport (MBJS) für die Bereitstellung der Daten.

Funding Open Access funding enabled and organized by Projekt DEAL.

Open Access Dieser Artikel wird unter der Creative Commons Namensnennung 4.0 International Lizenz veröffentlicht, welche die Nutzung, Vervielfältigung, Bearbeitung, Verbreitung und Wiedergabe in jeglichem Medium und Format erlaubt, sofern Sie den/die ursprünglichen Autor(en) und die Quelle ordnungsgemäß nennen, einen Link zur Creative Commons Lizenz beifügen und angeben, ob Änderungen vorgenommen wurden.

Die in diesem Artikel enthaltenen Bilder und sonstiges Drittmaterial unterliegen ebenfalls der genannten Creative Commons Lizenz, sofern sich aus der Abbildungslegende nichts anderes ergibt. Sofern das betreffende Material nicht unter der genannten Creative Commons Lizenz steht und die betreffende Handlung nicht nach gesetzlichen Vorschriften erlaubt ist, ist für die oben aufgeführten Weiterverwendungen des Materials die Einwilligung des jeweiligen Rechteinhabers einzuholen.

Weitere Details zur Lizenz entnehmen Sie bitte der Lizenzinformation auf http://creativecommons.org/ licenses/by/4.0/deed.de.

\section{Literatur}

van Ackeren, I., Binnewies, C., Clausen, M., Demski, D., Dormann, C., Koch, A. R., Laier, B., Preisendörfer, P., Preuße, D., Rosenbusch, C., Schmidt, U., Stump, M., \& Zlatkin-Troitschanskaia, O. (2013). Welche Wissensbestände nutzen Schulen im Kontext von Schulentwicklung? Theoretische Konzepte und erste deskriptive Befunde des EviS-Verbundprojektes im Überblick. Die Deutsche Schule, 13(Beiheft 12), 51-73.

Altrichter, H., \& Merki, M. K. (2016). Steuerung der Entwicklung des Schulwesens. In H. Altrichter \& K. Maag Merki (Hrsg.), Handbuch Neue Steuerung im Schulsystem (2. Aufl. S. 1-27). Wiesbaden: Springer VS.

Altrichter, H., Rürup, M., \& Schuchart, C. (2016). Schulautonomie und die Folgen. In H. Altrichter \& K. Maag Merki (Hrsg.), Handbuch Neue Steuerung im Schulsystem (2. Aufl. S. 107-149). Wiesbaden: Springer VS.

Amt für Statistik Berlin-Brandenburg (2015). Statistischer Bericht B I 1 -j/14. Allgemeinbildende Schulen im Land Brandenburg Schuljahr 2014/2015. Potsdam: Amt für Statistik Berlin-Brandenburg.

Appius, S., Steger Vogt, E., Kansteiner-Schänzlin, K., \& Bach-Blattner, T. (2012). Personalentwicklung an Schulen. Eine Bestandsaufnahme aus Sicht deutscher und schweizerischer Schulleitungen. Empirische Pädagogik, 26(1), 123-141.

Bach, A., Böhnke, A., \& Thiel, F. (2020). Improving instructional competencies through individualized staff development and teacher collaboration in German schools. International Journal of Educational Management, 34(8), 1289-1302. https://doi.org/10.1108/IJEM-08-2019-0294.

Basold, K. (2010). Zur Entwicklung von Einzelschulen durch Schulinterne Lehrerfortbildung. Eine kritische Auseinandersetzung auf der Grundlage einer Analyse von Berichten niedersächsischer Hauptund Realschulen. Dissertation. Hamburg: disserta.

Baumert, J., \& Kunter, M. (2006). Stichwort: Professionelle Kompetenz von Lehrkräften. Zeitschrift für Erziehungswissenschaft, 9(4), 469-520.

Baumert, J., Maaz, K., Stanat, P., \& Watermann, R. (2009). Schulkomposition oder Institution - was zählt? Schulstrukturen und die Entstehung schulformspezifischer Entwicklungsverläufe. Die Deutsche Schule, 101(1), 33-46.

Brauckmann, S., \& Schwarz, A. (2015). No time to manage? The trade-off between relevant tasks and actual priorities of school leaders in Germany. International Journal of Educational Management, 29(6), 749-765.

Bühl, A. (2006). SPSS 14: Einführung in die moderne Datenanalyse (10. Aufl.). München: Pearson Studium.

Cohen, J. (1988). Statistical power analysis for the behavioral sciences (2. Aufl.). Hillsdale: Lawrence Erlbaum.

Darling-Hammond, L., Hyler, M.E., \& Gardner, M. (2017). Effective teacher professional development. Palo Alto: Learning Policy Institute. 
Daschner, P. (2019). Intransparent, unterentwickelt, unterfinanziert. Befunde einer Bestandsaufnahme der Lehrerfortbildung in Deutschland. In N. McElvany, F. Schwabe, W. Bos \& H. G. Holtappels (Hrsg.), IFS-Bildungsdialoge 1. Aufl. Lehrerbildung - Potentiale und Herausforderungen in den drei Phasen, (Bd. 3, S. 71-91). Münster: Waxmann.

Dedering, K. (2012). Steuerung und Schulentwicklung. Bestandsaufnahme und Theorieperspektiven. Wiesbaden: Springer VS.

Deutscher Verein zur Förderung der Lehrerinnen und Lehrerfortbildung e. V. (DVLfB) (Hrsg.). (2018). Recherchen für eine Bestandsaufnahme der Lehrkräftefortbildung in Deutschland. Ergebnisse des Projektes Qualitätsentwicklung in der der Lehrkräftefortbildung. Teil 1. forum Lehrerfortbildung (47).

Ditton, H. (2000). Qualitätskontrolle und -sicherung in Schule und Unterricht: Eine Übersicht über den empirischen Forschungsstand. Zeitschrift für Pädagogik, 41, 73-92.

Ditton, H., \& Müller, A. (2015). Schulqualität. In H. Reinders (Hrsg.), Empirische Bildungsforschung. Gegenstandsbereiche (S. 121-134). Wiesbaden: Springer VS.

Felbrich, A., Müller, C., \& Blömeke, S. (2008). Lerngelegenheiten in der Lehrerausbildung. In S. Blömeke, G. Kaiser \& R. Lehmann (Hrsg.), Professionelle Kompetenz angehender Lehrerinnen und Lehrer. Wissen, Überzeugungen und Lerngelegenheiten deutscher Mathematikstudierender und-referendare. Erste Ergebnisse zur Wirksamkeit der Lehrerausbildung (S. 327-362). Münster: Waxmann.

Fischer, S. (2017). Zur Nachhaltigkeit von Lehrerweiterbildung in der Schweiz. Eine explorative Studie aus der Perspektive von Lehrkräften. Zeitschrift für Weiterbildungsforschung, 40, 241-259.

KMK (2000). Aufgaben von Lehrerinnen und Lehrern heute - Fachleute für das Lernen. Berlin: KMK.

KMK (2004). Bildungsstandards der Kultusministerkonferenz. Erläuterungen zur Konzeption und Entwicklung. http://www.kmk.org/fileadmin/veroeffentlichungen_beschluesse/2004/2004_12_16Bildungsstandards-Konzeption-Entwicklung.pdf. Zugegriffen: 1. Okt. 2020.

KMK (2017). Lehrkräftefortbildung in den Ländern. Berlin: KMK.

Kuschel, J., Richter, D., \& Lazarides, R. (2020). Wie relevant ist die gesetzliche Fortbildungsverpflichtung für Lehrkräfte? Eine empirische Untersuchung zur Fortbildungsteilnahme in verschiedenen deutschen Bundesländern. Zeitschrift für Bildungsforschung, 10, 211-229. https://doi.org/10.1007/s35834-02000274-3.

Kutner, M.H. (2005). Applied linear statistical models. Boston: McGraw-Hill Irwin.

Landesregierung Brandenburg (2015). Gemeinsames Lernen in der Schule gemäß Beschluss des Landtages vom 17. Dezember 2015 ,Inklusion im Bildungssystem Brandenburg weiter kontinuierlich vorantreiben “. Potsdam: Landtag.

Lucksnat, C., Richter, E., Klusmann, U., Kunter, M., \& Richter, D. (2020). Unterschiedliche Wege ins Lehramt - unterschiedliche Kompetenzen? Ein Vergleich von Quereinsteigern und traditionell ausgebildeten Lehramtsanwärtern im Vorbereitungsdienst. Zeitschrift für pädagogische Psychologie, 40, 1-16. https://doi.org/10.1024/1010-0652/a000280.

Maier, U., Metz, K., Bohl, T., Kleinknecht, M., \& Schymala, M. (2012). Vergleichsarbeiten als Instrument der datenbasierten Schul- und Unterrichtsentwicklung in Gymnasien. In A. Wacker, U. Maier \& J. Wissinger (Hrsg.), Schul- und Unterrichtsreform durch ergebnisorientierte Steuerung. Empirische Befunde und forschungsmethodische Implikationen (S. 197-224). Wiesbaden: VS. https://doi. org/10.1007/978-3-531-94183-7_9.

Mentzel, W. (2001). Personalentwicklung. Erfolgreich motivieren, fördern und weiterbilden. München: Dt. Taschenbuch-Verlag.

Meyer, A., Richter, D., Marx, A., \& Hartung-Beck, V. (2019). Welche Aufgaben haben Schulleitungen heute? Eine Analyse von Schulleitungsaufgaben im innerdeutschen Vergleich. Zeitschrift für Bildungsverwaltung, 35(2), 23-44.

Missal, S. (2019). Erfolgreiche Konzepte der Weiterbildung von Lehrkräften. Zur Fortbildung von Lehrkräften und Führungskräften im schulischen Kontext in Brandenburg. In N. McElvany, F. Schwabe, W. Bos \& H.G. Holtappels (Hrsg.), IFS-Bildungsdialoge 1. Aufl. Lehrerbildung - Potentiale und Herausforderungen in den drei Phasen, (Bd. 3, S. 109-126). Münster: Waxmann.

Pasternack, P., Baumgarth, B., Burkhardt, A., Paschke, S., \& Thielemann, N. (2017). Drei Phasen. Die Debatte zur Qualitätsentwicklung in der Lehrer_innenbildung. GEW-Materialien aus Hochschule und Forschung, Bd. 124. Bielefeld: wbv.

Richter, D. (2016). Lehrerinnen und Lehrer lernen. Fort- und Weiterbildung im Lehrerberuf. In M. Rothland (Hrsg.), Beruf Lehrer/Lehrerin. Ein Studienbuch. Münster: Waxmann.

Richter, D., Becker, B., Hoffmann, L., Busse, J., \& Stanat, P. (2019). Aspekte der Aus- und Fortbildung von Lehrkräften im Fach Mathematik und in den naturwissenschaftlichen Fächern. In P. Stanat, S. Schipolowski, N. Mahler, S. Weirich \& S. Henschel (Hrsg.), IQB-Bildungstrend 2018. Mathematische 
und naturwissenschaftliche Kompetenzen am Ende der Sekundarstufe I im zweiten Ländervergleich (S. 385-410). Münster, New York: Waxmann.

Richter, E., \& Richter, D. (2020). Fort- und Weiterbildung von Lehrpersonen. In C. Cramer, J. König \& M. Rothland (Hrsg.), Handbuch Lehrerinnen- und Lehrerbildung (S. 345-354). Bad Heilbrunn: Julius Klinkhardt. https://doi.org/10.35468/hblb2020-040.

Richter, E., Marx, A., Huang, Y., \& Richter, D. (2020). Zeiten zum beruflichen Lernen: Eine empirische Untersuchung zum Zeitpunkt und der Dauer von Fortbildungsangeboten für Lehrkräfte. Zeitschrift für Erziehungswissenschaft, 23(1), 145-173. https://doi.org/10.1007/s11618-019-00924-x.

Rzejak, D., \& Lipowsky, F. (2020). Fort- und Weiterbildung von Lehrpersonen. In C. Cramer, J. König \& M. Rothland (Hrsg.), Handbuch Lehrerinnen- und Lehrerbildung (S. 644-652). Bad Heilbrunn: Julius Klinkhardt.

Steger Vogt, E., \& Kabitz, S. (2014). Die Führungsakzeptanz der Personalentwicklung durch die Lehrpersonen. In E. Steger Vogt, K. Kansteiner \& M. Pfeifer (Hrsg.), Gelingende Personalentwicklung in der Schule (S. 133-146). Innsbruck: StudienVerlag.

Thillmann, K., Brauckmann, S., Herrmann, C., \& Thiel, F. (2015). Praxis schulischer Personalentwicklung unter den Bedingungen der Neuen Steuerung. Empirische Befunde aus den Forschungsprojekten SHaRP und StABil. In H.J. Abs, T. Brüsemeister, M. Schemmann \& J. Wissinger (Hrsg.), Governance im Bildungssystem. Analysen zur Mehrebenenperspektive, Steuerung und Koordination (S. 195-228). Wiesbaden: Springer.

Tillmann, K.-J. (2011). Schultheorie, Schulentwicklung, Schulqualität. In H. Altrichter \& C. Helm (Hrsg.), Akteure \& Instrumente der Schulentwicklung. Professionswissen für Lehrerinnen und Lehrer (Bd. 7 , S. 37-57). Baltmannsweiler: Schneider Hohengehren.

Verwaltungsvorschriften über Anrechnungsstunden für Lehrkräfte (VV-Anrechnungsstunden) vom 30. Mai 2008. VV-AnrStd. 2008.

Verwaltungsvorschriften über die Fortbildung der Lehrkräfte an Schulen in öffentlicher Trägerschaft (VVLehrkräftefortbildung) vom 29. Apr. 2015. VV-LKFB. 2015.

Wenzel, H., \& Wesemann, M. (1990). Schulinterne Lehrerfortbildung: Begriffliche Klärungen, Abgrenzungen und Probleme. In H. Wenzel, M. Wesemann \& F. Bohnsack (Hrsg.), Studien zur Schulpädagogik und Didaktik. Schulinterne Lehrerfortbildung. Ihr Beitrag zu schulischer Selbstentwicklung, (Bd. 4, S. 24-40). Weinheim, Basel: Beltz.

Werding, E., \& Schinnenburg, H. (2016). Inklusion als Herausforderung für die Personalentwicklung. IstStand aus der Sicht von Fach- und Leitungskräften. In S. Maykus, A. Beck, G. Hensen, A. Lohmann, H. Schinnenburg, M. Walk, E. Werding \& S. Wiedebusch (Hrsg.), Inklusive Bildung in Kindertageseinrichtungen und Grundschulen. Empirische Befunde und Implikationen für die Praxis (S. 117-145). Weinheim, Basel: Beltz.

Wirtz, M., \& Kutschmann, M. (2007). Analyse der Beurteilerübereinstimmung für kategoriale Daten mittels Cohens Kappa und alternativer Maße. Die Rehabilitation, 46, 1-8. https://doi.org/10.1055/s2007-976535.

Wöhlbier, K., Groth, E., Tietz, S., Sobosczyk, T., Schwarz, H., Leu, S., \& Borrmann, A. (2019). Ergebnisse aus dem Prozess der Werkstatt schulentwicklung.digital 2018/19. Mikrofortbildungen. https://www. forumbd.de/publikationen/praxisleitfaden-mikrofortbildungen/. Zugegriffen: 06.05.2021.

Wurster, S., Richter, D., \& Lenski, A.E. (2017). Datenbasierte Unterrichtsentwicklung und ihr Zusammenhang zur Schülerleistung. Zeitschrift für Erziehungswissenschaft, 20, 628-650. https://doi.org/ 10.1007/s11618-017-0759-x. 